\title{
Self-consistent atmosphere modeling with cloud formation for low-mass stars and exoplanets
}

\author{
Diana Juncher ${ }^{1}$, Uffe G. Jørgensen ${ }^{1}$, and Christiane Helling ${ }^{2}$
${ }^{1}$ Niels Bohr Institute \& Centre for Star and Planet Formation, University of Copenhagen, Øster Voldgade 5, 1350 Copenhagen, Denmark e-mail: diana@nbi.dk
2 Centre for Exoplanet Science, SUPA, School of Physics and Astronomy, University of St Andrews, North Haugh, St Andrews, Fife, KY16 9SS, UK

Received 30 October 2016 / Accepted 16 August 2017

\begin{abstract}
Context. Low-mass stars and extrasolar planets have ultra-cool atmospheres where a rich chemistry occurs and clouds form. The increasing amount of spectroscopic observations for extrasolar planets requires self-consistent model atmosphere simulations to consistently include the formation processes that determine cloud formation and their feedback onto the atmosphere.

Aims. Our aim is to complement the MARCS model atmosphere suit with simulations applicable to low-mass stars and exoplanets in preparation of E-ELT, JWST, PLATO and other upcoming facilities.

Methods. The MARCS code calculates stellar atmosphere models, providing self-consistent solutions of the radiative transfer and the atmospheric structure and chemistry. We combine MARCS with a kinetic model that describes cloud formation in ultra-cool atmospheres (seed formation, growth/evaporation, gravitational settling, convective mixing, element depletion).

Results. We present a small grid of self-consistently calculated atmosphere models for $T_{\text {eff }}=2000-3000 \mathrm{~K}$ with solar initial abundances and $\log (g)=4.5$. Cloud formation in stellar and sub-stellar atmospheres appears for $T_{\text {eff }}<2700 \mathrm{~K}$ and has a significant effect on the structure and the spectrum of the atmosphere for $T_{\text {eff }}<2400 \mathrm{~K}$. We have compared the synthetic spectra of our models with observed spectra and found that they fit the spectra of mid- to late-type M-dwarfs and early-type L-dwarfs well. The geometrical extension of the atmospheres (at $\tau=1$ ) changes with wavelength resulting in a flux variation of $\sim 10 \%$. This translates into a change in geometrical extension of the atmosphere of about $50 \mathrm{~km}$, which is the quantitative basis for exoplanetary transit spectroscopy. We also test DRIFT-MARCS for an example exoplanet and demonstrate that our simulations reproduce the Spitzer observations for WASP-19b rather well for $T_{\text {eff }}=2600 \mathrm{~K}, \log (g)=3.2$ and solar abundances. Our model points at an exoplanet with a deep cloud-free atmosphere with a substantial day-night energy transport and no temperature inversion.
\end{abstract}

Key words. astrochemistry - radiative transfer - methods: numerical - stars: atmospheres - stars: low-mass - brown dwarfs

\section{Introduction}

The atmospheres of late-type M-dwarf stars and brown dwarfs collectively referred to as ultra-cool dwarfs - and planets have low-enough temperatures for clouds to form. Cloud formation increases the total atmospheric opacity but also affects the local gas phase by element depletion. A strong effect on the structure of their atmospheres results, and hence self-consistent inclusion of cloud formation is critical for correctly inferring the physical structure and chemical composition of these objects from observed spectra. The same physics and considerations apply to the atmospheres of the bulk of known exoplanets, and the present paper is therefore our first paper in a series of planned papers to describe self-consistent modeling of exoplanetary atmospheres as a tool for interpreting forthcoming high-quality spectra of exoplanets that will become available with the next generation instruments during the coming years.

The presence of cloud formation in ultra-cool dwarf atmospheres was first proposed by Lunine et al. (1986) from the comparison of temperature-pressure profiles of brown dwarf atmosphere models with the condensation curves of refractory materials such as iron, sodium-aluminum silicates, and magnesium silicates. A decade later, Tsuji et al. (1996) presented the first cloud modeling results for brown dwarfs, showing how the high opacity of dust particles can produce a noticeable effect in the observed spectrum. Tsuji et al. (1996) also suggested that cloud formation should be considered for all objects with $T_{\text {eff }}<$ $2800 \mathrm{~K}$ and should therefore also be included in the models of late-type $\mathrm{M}$ dwarf atmospheres.

Modeling cloud formation is a complex problem involving different coupled processes that depend on a wide range of physical and chemical parameters. Many of the early models of cloudy atmospheres (e.g., Rossow 1978; Lewis 1969; Carlson et al. 1988; Lunine et al. 1986; Burrows et al. 1989; and Tsuji et al. 1996) were able to reproduce basic features of ultracool dwarfs by simply turning on or off the opacity of dust in the atmosphere at its chemical equilibrium temperature-pressure location. Over the years the models have grown more detailed and more realistic, and today several independent groups are working on complex models that represent clouds in atmosphere models using different strategies. Some are based on practical considerations (Tsuji 2001; Barman et al. 2011; Burrows et al. 2006), while others are inspired by measurements of the atmospheres of the planets in our solar system (Allard et al. 2001; Cooper et al. 2003), terrestrial cloud formation (Ackerman \& Marley 2001), or kinetic dust-formation modeling in asymptotic giant branch stars (AGB; Helling et al. 2001; Woitke \& Helling 2003, 2004). A detailed comparison of a selection of these can be found in Helling et al. (2008a). 
In this paper we present an extension of the MARCS code (Gustafsson 1971; Jørgensen et al. 1992; Gustafsson et al. 2008; Van Eck et al. 2017) that has so far been used extensively for modeling atmospheres of cool stars (Lambert et al. 1986; Plez 1992; Aringer et al. 1997), including abundance analyses (e.g., Blackwell et al. 1995; Matrozis et al. 2013; Nissen et al. 2014; Hill et al. 2017; Siqueira-Mello et al. 2016), $\mathrm{H}_{2} \mathrm{O}$ detections (Ryde et al. 2002; Aringer et al. 2002), microdiamonds in carbon stars (Andersen \& Jørgensen 1995), and instrument calibration (Decin et al. 2003; Decin \& Eriksson 2007). MARCS has also been used to study cool, helium-rich white dwarfs (Jørgensen et al. 2000), R Coronae Borealis stars (Asplund et al. 2000), and to determine fundamental properties of gamma-ray burst (GRB) progenitors (Groh et al. 2013). While the radiativetransfer treatment of MARCS has inspired time-dependent carbon-rich models for dust-forming AGB stars (Höfner et al. 1998), their lower-mass counterparts, that is, late type M-dwarfs and brown dwarfs with clouds, have not been addressed by the MARCS community so far. This paper presents MARCS model atmosphere simulations which include a detailed modeling of cloud formation, by self-consistently solving the radiative transfer and gas-phase chemistry in the scheme of MARCs together with the seed formation, growth/evaporation of cloud particles, element conservation and gravitational settling in the scheme of DRIFT. In this way the radiative and chemical feedback on the atmosphere due to cloud formation is fully taken into account. Section 2 summarizes our approach, including tables of input properties. We present our results for a grid of DRIFT-MARCS model atmosphere simulations applicable to solar-metallicity Mdwarfs and brown dwarfs $\left(T_{\text {eff }}=2000-3000 \mathrm{~K}, \log (g)=4.5\right.$; Sect. 3). These models represent an extension of the MARCs code with respect to the updated gas-phase opacity data and the modeling of cloud formation. They also offer a new alternative to the DRIFT-PHOENIX models. We compare the synthetic spectra resulting from our atmosphere simulations with observed spectra of mid- to late-type M-dwarfs, early to mid-type L-dwarfs, and an example giant gas planet WASP-19b in Sect. 4. Section 5 discusses the effect of porosity in cloud particles. Appendix B provides additional details about the gas species contributing to the synthetic spectra.

\section{Approach}

Two well-tested codes are combined to enable hands-on atmosphere simulations for ultra-cool, cloud-forming objects. DRIFT, the cloud formation module, has been applied to investigate cloud structures in brown dwarfs and extrasolar planets from first principles (e.g., Helling et al. 2008b; Street et al. 2015; Helling et al. 2016). MARCS has been applied to a large number of atmosphere problems (Sect. 1). We follow a similar strategy as in Witte et al. (2009) in combining the two codes. In the following, we provide a summery of the two codes, the opacity data used, and the methodology for running the combined codes.

\subsection{MARCS}

The code: the MARCS code was introduced in the early 1970s by Gustafsson et al. (1975) and has since then been developed in step with the advancement of computer power and available physical data. The most recent general grid of MARCs models was published by Gustafsson et al. (2008) and contains about 50000 state-of-the-art stellar atmosphere models extending from late A-type to early M-type stars - from dwarfs to supergiants for varying metallicities and C/O-ratios. This version of MARCs
Table 1. Sources of data for continuum opacities.

\begin{tabular}{lll}
\hline \hline Ion & Process & Reference \\
\hline $\mathrm{H}^{-}$ & b-f & Doughty et al. (1966) \\
$\mathrm{H}^{-}$ & f-f & Doughty \& Fraser (1966) \\
$\mathrm{H} I$ & b-f, f-f & Karzas \& Latter (1961) \\
$\mathrm{H}$ I+HI & CIA & Doyle (1968) \\
$\mathrm{H}_{2}^{-}$ & f-f & Somerville (1964) \\
$\mathrm{H}_{2}^{+}$ & f-f & Mihalas (1965) \\
$\mathrm{He}^{-}$ & f-f & Somerville (1965), John (1967) \\
$\mathrm{HeI}_{\mathrm{I}}$ & $\mathrm{f}-\mathrm{f}$ & Peach (1970) \\
$\mathrm{CI}$, & $\mathrm{f}-\mathrm{f}$ & Peach (1970) \\
$\mathrm{MgI}$ & $\mathrm{f}-\mathrm{f}$ & Peach (1970) \\
$\mathrm{AlI}$, & f-f & Peach (1970) \\
$\mathrm{Si}$ & f-f & Peach (1970) \\
$\mathrm{e}^{-}$ & Scattering & Mihalas (1978) \\
$\mathrm{HI}$ & Scattering & Dalgarno, quoted by Kurucz (1970) \\
\hline
\end{tabular}

Notes. "b-f" and "f-f" denote bound-free and free-free processes, respectively. CIA stands for collision induced absorption.

is very similar to our version, and details of the implementation of hydrostatic equilibrium, radiative transfer, convection and mixing length can be found in Gustafsson et al. (2008). For the equilibrium calculations we use a version of Tsuji's program (Tsuji 1964) implemented by Helling et al. (1996), and updated further for the present work.

Input data: the chemical equilibrium calculations in MARCS are based on 38 atoms and 210 molecules (see Appendix A). We have adopted the chemical composition of the Sun as reported by Grevesse et al. (2007) for all our models. For the atoms and ions we use the internal partition function data from Irwin (1981) to calculate the equilibrium constants. For the molecules we use the Gibbs free energy data from Tsuji (1973), Burrows \& Sharp (1999) and Burrows et al. (2005) to calculate the equilibrium constants.

We calculate the continuum absorption for about a dozen ions, electron scattering and Rayleigh scattering by Hi (Table 1). The line opacities for atoms and ions were updated by Popovas (2014) with atomic line data from VALD-3 (Kupka et al. 2011). The line opacities for molecules were updated to include the 24 molecules and molecular pairs listed in Table 2. We sampled all line opacities using the Opacity Sampling method with a resolution of $R=\lambda / \Delta \lambda=20000$ in the wavelength range $0.125-25 \mu \mathrm{m}$.

As described in Gustafsson et al. (2008), the convection in MARCS is handled using the mixing length method, where the convective energy flux can be calculated as a function of the mixing length $l$. The value of $l$ is based on empirical calibrations of stellar interior models and is thus not theoretically derived. It is often expressed as a product of the mixing length parameter $\alpha$ and the scale height. For cool stars and brown dwarfs $\alpha \approx 2$ (Ludwig et al. 2002) and this is the value we adopt for our models.

\subsection{DRIFT}

The code: the DRIFT code models cloud formation by considering each of the involved physical and chemical processes in detail. The formation of seed particles and the subsequent growth or evaporation of dust grains are described by modified 
Table 2. Molecular line transitions and their sources.

\begin{tabular}{|c|c|c|}
\hline Molecule & Transitions & Reference \\
\hline \multicolumn{3}{|l|}{ Hydrides } \\
\hline $\mathrm{LiH}$ & vib-rot & Coppola et al. (2011) \\
\hline \multirow{2}{*}{$\mathrm{MgH}$} & vib-rot & Yadin et al. (2012) \\
\hline & A-X, B'-X & GharibNezhad et al. (2013) \\
\hline $\mathrm{SiH}$ & $\mathrm{A}-\mathrm{X}$ & Kurucz (2011) \\
\hline \multirow{2}{*}{$\mathrm{CaH}$} & vib-rot & Yadin et al. (2012) \\
\hline & A-X, B-X, C-X, D-X, E-X & Weck et al. (2003) \\
\hline $\mathrm{TiH}$ & A-X, B-X & Burrows et al. (2005) \\
\hline $\mathrm{CrH}$ & A-X & Burrows et al. (2002) \\
\hline $\mathrm{FeH}$ & F-X & Wende et al. (2010) \\
\hline $\mathrm{CH}$ & vib-rot, A-X, B-X, C-X & Masseron et al. (2014) \\
\hline \multirow[t]{2}{*}{$\mathrm{NH}$} & vib-rot & Brooke et al. (2014a) \\
\hline & A-X, A-C & Kurucz (2011) \\
\hline $\mathrm{OH}$ & vib-rot, A-X & Kurucz (2011) \\
\hline \multicolumn{3}{|l|}{ Oxides } \\
\hline \multirow[t]{2}{*}{$\mathrm{SiO}$} & vib-rot & Barton et al. (2013) \\
\hline & A-X, E-X & Kurucz (2011) \\
\hline $\mathrm{TiO}$ & $\begin{array}{l}\text { A-X, B-X, C-X, E-X } \\
\text { c-a, b-a, b-d, f-a }\end{array}$ & Schwenke (1998) \\
\hline VO & A-X, B-X, C-X & Kurucz (2011) \\
\hline $\mathrm{ZrO}$ & $\begin{array}{l}\text { B-A, B-X, C-X, E-A } \\
\text { b-a, d-a, e-a, f-a }\end{array}$ & Plez et al. (2003) \\
\hline $\mathrm{CO}$ & vib-rot, A-X & Kurucz (2011) \\
\hline NO & vib-rot & Rothman et al. (2010) \\
\hline $\mathrm{H}_{2} \mathrm{O}$ & vib-rot & Jørgensen et al. (2001) \\
\hline \multicolumn{3}{|l|}{ Other } \\
\hline $\mathrm{H}_{2}, \mathrm{HD}$ & vib-rot, quad, B-X, C-X & Kurucz (2011) \\
\hline \multirow{2}{*}{$\mathrm{C}_{2}$} & A-X, b-a, E-A & Kurucz (2011) \\
\hline & d-a & Brooke et al. (2013) \\
\hline $\mathrm{CN}$ & vib-rot, A-X, B-X & Brooke et al. (2014b) \\
\hline & vib-rot & Rothman et al. (2010) \\
\hline \multirow{2}{*}{$\mathrm{HCN}$} & vib-rot & Harris et al. (2006) \\
\hline & & Harris et al. (2008) \\
\hline $\mathrm{H}_{2}-\mathrm{H}_{2}$ & CIA & Borysow et al. (2001) \\
\hline $\mathrm{H}_{2}-\mathrm{He}$ & CIA & Jørgensen et al. (2000) \\
\hline
\end{tabular}

classical nucleation theory and the moment method, respectively (Gail \& Sedlmayr 1988; Dominik et al. 1993; Lee et al. 2015). The initial model equations where extended to describe the growth/evaporation of particles of mixed material composition as required in particular for oxygen-rich atmospheres (Helling \& Woitke 2006; Helling et al. 2008b). This is coupled to the effects of gravitational settling, convective mixing and element depletion via a system of partial differential equations (Woitke \& Helling 2003, 2004; Helling \& Woitke 2006).

The convection in ultra-cool dwarfs allows for the upwards transport and subsequent diffusion of the non-depleted gas from the interior of the dwarf. This convective mixing can be extended into the upper, radiative atmosphere via overshooting, thereby facilitating a replenishment of the depleted gas above the cloud base, maintaining the dust cycle. The DRIFT code models overshooting by assuming an exponential decrease of the mass exchange frequency above the radiative zone (Eq. (9) in Woitke \& Helling 2004, with $\beta=2.2$ and $\left.\tau_{\text {mix }}^{\text {min }}=2 /\left(H_{\mathrm{p}} v_{\mathrm{c}}\right)\right)$.

We consider seven growth species $\left(\mathrm{TiO}_{2}[\mathrm{~s}], \mathrm{MgSiO}_{4}[\mathrm{~s}]\right.$, $\mathrm{SiO}_{2}[\mathrm{~s}], \mathrm{Fe}[\mathrm{s}], \mathrm{Al}_{2} \mathrm{O}_{3}[\mathrm{~s}], \mathrm{MgO}[\mathrm{s}]$ and $\mathrm{MgSiO}_{3}$ [s]) to make this initial implementation as simple as possible. We include 32 chemical surface reactions which is a subset of reactions of Helling et al. (2008b) for the respective materials. For $\mathrm{TiO}_{2}$ we use the data from Woitke \& Helling (2003) to calculate the saturation vapor pressure at different temperatures. For the remaining condensates we use the data from Sharp \& Huebner (1990).
Table 3. References for $n$ and $k$ optical constants of the condensates.

\begin{tabular}{ll}
\hline \hline Solid species & Reference \\
\hline $\mathrm{TiO}_{2}[\mathrm{~s}]$ & Ribarsky in Palik (1985) \\
$\mathrm{MgSiO}_{4}[\mathrm{~s}]$ & Jäger et al. (2003) \\
$\mathrm{SiO}_{2}[\mathrm{~s}]$ & Posch et al. (2003) \\
$\mathrm{Fe}[\mathrm{s}]$ & Posch et al. (2003) \\
$\mathrm{Al}_{2} \mathrm{O}_{3}[\mathrm{~s}]$ & Zeidler et al. (2013) \\
$\left.\mathrm{MgO}^{\mathrm{s}}\right]$ & Roessler \& Huffman in Palik (1985) \\
$\mathrm{MgSiO}_{3}[\mathrm{~s}]$ & Dorschner et al. (1995) \\
\hline
\end{tabular}

Dust opacity: DRIFT calculates the vertical distribution of the clouds as well as the size and composition of their cloud particles, but to assess how the opacity of the clouds affect the structure we also need to calculate the absorption and scattering of the dust grains.

From the information provided by DRIFT about a specific cloud particle size and the volume of each of its components, we can use the Bruggeman equations (Bruggeman 1935) to calculate its effective index of refraction, assuming that the dust grain is compact and its components are randomly mixed. This allows us to treat the dust grain as a homogeneous particle, the properties of its components combining to generate effective properties of the whole particle itself.

Because the size of the dust grains are typically of the same order as the wavelength of the starlight, we cannot use the Rayleigh or geometrical approximations to describe how they interact with the light. Instead we have to use full Mie Theory (Mie 1908; Bohren \& Huffman 1983) for a complete description of how electromagnetic plane weaves are absorbed and scattered by homogeneous spherical particles. This, of course, also requires the assumption that the dust grains are spherical.

Input data: the sources of the optical constants used to calculate the effective index of refraction of the mixed dust particles are given in Table 3 . Most of the data covers the wavelength range 1.25-25 $\mu \mathrm{m}$, only the data for $\mathrm{Al}_{2} \mathrm{O}_{3}[\mathrm{~s}]$ and $\mathrm{MgSiO}_{3}[\mathrm{~s}]$ had to be extrapolated down to the lowest considered wavelength. We did this by freezing the optical constants from the first known wavelength points.

\subsection{Merging MARCS with DRIFT}

In order to calculate the details of the cloud layers in an atmosphere, DRIFT needs information about the $\left(T_{\mathrm{g}}, P_{\mathrm{g}}\right)$-structure, chemical composition and convection of the atmosphere. Similarly, MARCS needs information about the size and composition of the cloud particles as well as the depletion of elements to calculate the effects of clouds in the atmosphere. We manage this data exchange between MARCS and DRIFT through input and output files containing the information listed in Table 4.

Changes to the MARCS code: in previous versions of the MARCS code the element abundances have been considered constant throughout the atmosphere. Since diffusion of atoms is a very slow process that only becomes dominant in stars hotter than $T_{\text {eff }} \approx 11500 \mathrm{~K}$ (Hui-Bon-Hoa et al. 2000), this is usually an excellent approximation and especially so for late-type stars, where the deep convective envelopes keeps the gas well mixed. However, in ultra-cool dwarfs the dust formation will cause a depletion of elements in the top layers where the dust 
Table 4. Data exchanged between MARCS and DRIFT.

\begin{tabular}{rl|cl}
\hline \hline MARCS to DRIFT & & & DRIFT to MARCS \\
\hline Layer height & $z$ & $a(z)$ & Average grain size \\
Gas temperature & $T(z)$ & & \\
Gas pressure & $P(z)$ & $V_{i}(z)$ & Average grain \\
Gas density & $\rho(z)$ & & Volume fractions \\
Gravitational acceleration & $g(z)$ & & \\
Convection velocity & $v_{c}(z)$ & $\epsilon_{i}(z)$ & Depleted element \\
Mixing length parameter & $l$ & & abundances \\
Initial element abundances & $\epsilon_{i}^{0}$ & & \\
\hline
\end{tabular}

grains form, and a corresponding augmentation of elements in the layers where the dust grains evaporates. We have therefore expanded the initial one-dimensional array containing element abundances with an extra dimension to account for their depth dependence.

The coolest models of Gustafsson et al. (2008) start at a Rosseland optical depth of $\log (\tau)=-5$, ends at $\log (\tau)=2$, and have a resolution of $\Delta \log (\tau)=0.2$. This is appropriate for cloud free models, and extending the atmospheres or increasing the resolution would have little effect on the computed model. But since cloud formation can set in at much lower optical depths than $\log (\tau)=-5$ we found it necessary to extend our models out to $\log (\tau)=-10$. Furthermore, we also increased the resolution in the upper layers to $\Delta \log (\tau)=0.15$ to accommodate the potentially rapid changes over short distances that cloud formation can cause.

Changes to the DRIFT code: the changes to the DRIFT code were minimal, consisting only of the addition of a new routine to handle the communication with MARCS.

Running DRIFT-MARcs: while MARCs needs to know the element depletion and dust opacity before it can solve the radiative transfer equation, DRIFT needs to know the convection speed which is calculated by MARCS as it solves the radiative transfer equation. At first glance it seems like we are in a deadlock, but the solution is actually relatively simple. If we start with a dustfree model of $T_{\text {eff }} \approx 3000 \mathrm{~K}$ and then proceed to gradually lower the effective temperature, iterating through MARCS and DRIFT for each step, the data exchange files will be updated in sync with the increasing dust formation. For this to work, the change in the effective temperature between each step had to be relatively small, about $T_{\mathrm{eff}}=10-50 \mathrm{~K}$ depending on the impact of the dust formation.

The dust-free version of MARCs will keep iterating over a model until the temperature corrections in all layers are below a given value, usually $T \leq 2 \mathrm{~K}$. However, if we allow MARCs to fulfill this convergence criterion every time we run DRIFT, we can easily end up in an endless loop with no convergence in sight. When DRIFT adds a layer of dust to the atmosphere, MARCS will heat the layers as a reaction to the increased opacity. In response, DRIFT will then reduce the amount of dust as the higher temperatures impede the dust formation. MARCs will of course react to the decreased opacity by cooling the layers again, and we are thus back where we started - or even further away. To avoid this, we only let MARCs iterate once between each call to DRIFT, and we limit the temperature correction to half of what the code suggests. This way we stop the overheating of the atmosphere and allow the dust formation to react to the temperature change before it becomes too large. When the

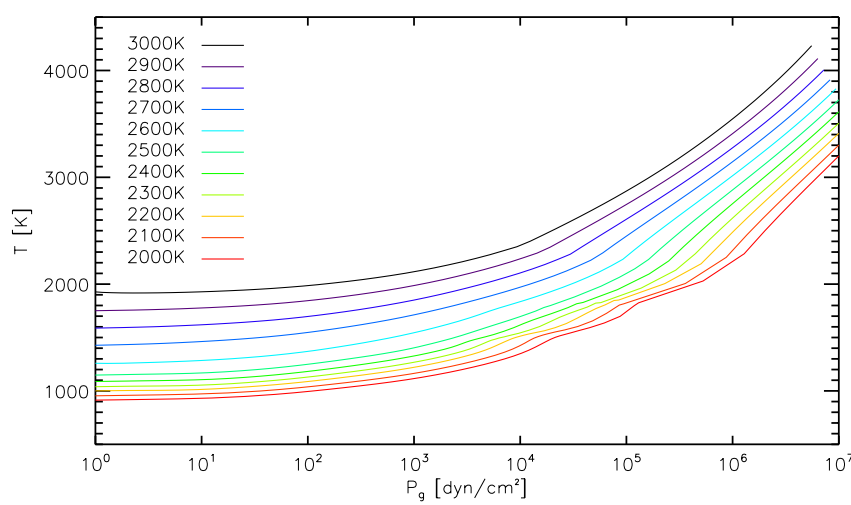

Fig. 1. $T-P_{\mathrm{g}}$ profiles for our grid of models with varying effective temperatures, $\log (g)=4.5$ and solar initial abundances.

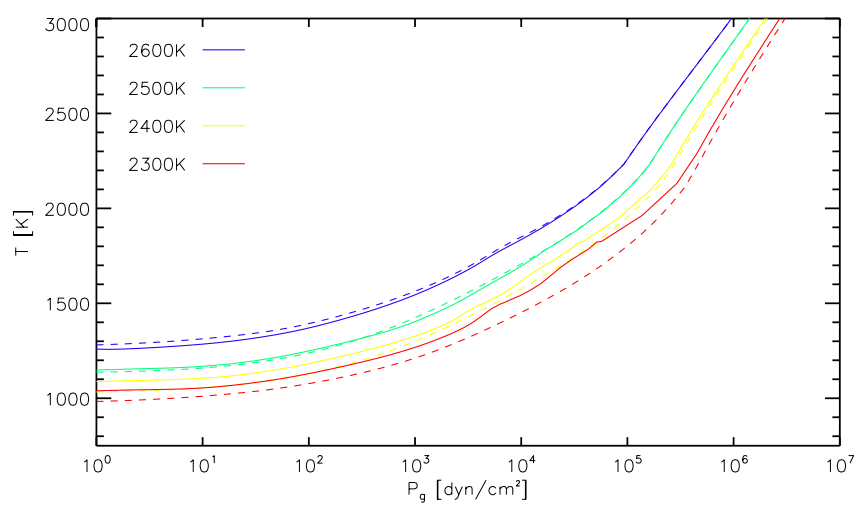

Fig. 2. $T-P_{\mathrm{g}}$ profiles for our grid of models with clouds (full drawn lines) compared to cloud-free models (dashed lines). All models have $\log (g)=4.5$ and solar initial abundances.

temperature correction is below $T \leq 10 \mathrm{~K}$ we consider the cloud layer stable and let MARCS converge fully without calling DRIFT again.

\section{Results}

We have created a small grid of models for late-type M-dwarfs and early L-type brown dwarfs with effective temperatures of $T_{\text {eff }}=2000-3000 \mathrm{~K}$ in steps of $T=100 \mathrm{~K}$. They all have solar initial abundances and a surface gravity of $\log (g)=4.5$. The specifics of these atmosphere models are discussed in the following.

\subsection{Atmosphere models}

We present the temperature-pressure profiles of our models in Fig. 1. Convection sets in at around $P_{\mathrm{g}}>10^{5} \mathrm{dyn} / \mathrm{cm}^{-1}$ and is the predominant mode of energy transport in the bottom layers of the atmosphere. In the upper layers the temperature gradient is very shallow.

In Fig. 2 we compare the temperature-pressure profiles of our cloud-forming models with models where the cloud forming has been switched off. For $T_{\text {eff }}<2700 \mathrm{~K}$ the temperature in the upper layers of the atmosphere is low enough for cloud formation to take place, but the effect is so small in the beginning that it barely affects the structure of the model. At $T_{\text {eff }}=2600 \mathrm{~K}$ the amount of dust formation has increased enough to cause a cooling effect in the outer layers. This happens because the depletion of the gas-phase elements that are now bound in dust 

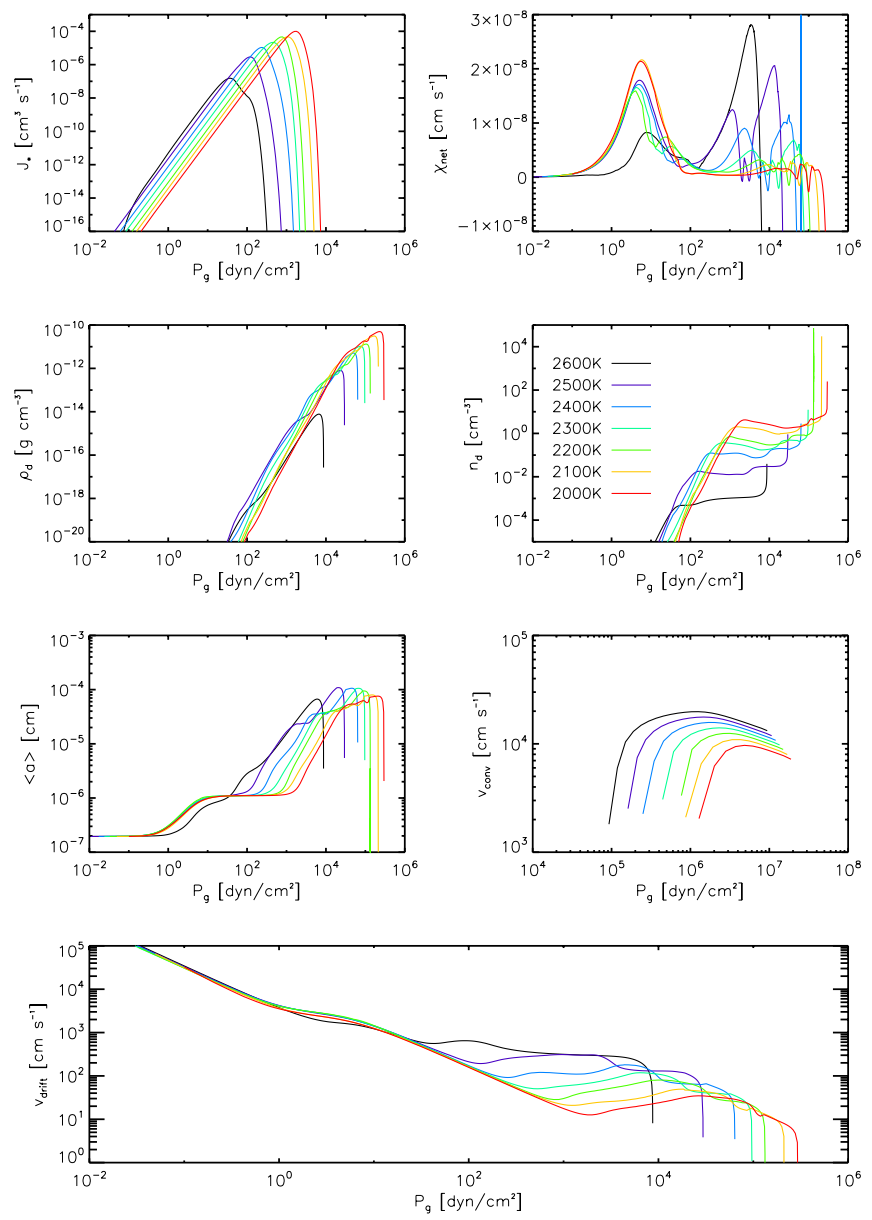

Fig. 3. Nucleation rate $J_{*}$, net growth rate $\chi_{\text {net }}$, mass density $\rho_{\mathrm{d}}$ and number density $n_{\mathrm{d}}$ of the dust grains, the mean grain size $\langle a\rangle$, the convective velocity $v_{\text {conv }}$, and the drift velocity $v_{\mathrm{d}}$ as a function of gas pressure. Color-coding is indicated in panel 4.

grains leads to a depletion of the gas-phase molecules that are made up of those specific elements. Although these molecules are a small fraction of the overall number of molecules, some of them are important absorbers and their depletion significantly reduces the opacity of the atmosphere. As long as the clouds are not substantial enough for their own opacity to compensate for the decreased molecular opacity, the affected layers will cool a little. At $T_{\text {eff }}=2600 \mathrm{~K}$ the upper layers cool about $10-20 \mathrm{~K}$, at $T_{\text {eff }}=2500 \mathrm{~K}$ the increase in cloud opacity more or less balances the decrease in molecular opacity, and at $T_{\text {eff }}=2400 \mathrm{~K}$ there is a clear heating of the upper layers caused by cloud formation. For successively cooler models the amount of heating in the upper layers increases correspondingly, and in the atmospheres of the coolest models the back-warming becomes more pronounced. The growing irregularities in the temperature at $P_{\mathrm{g}}=10^{4}-10^{6} \mathrm{dyn} / \mathrm{cm}^{2}$ coincide with the lower and densest part of the forming clouds (see Fig. 3).

There is a general tendency for the temperature irregularities to shift downwards for decreasing effective temperatures. This can be explained by a combination of the thermal stability temperature moving downward, with the withdrawal of the convection zone and a lower velocity of the convective cells, which makes the element replenishment less effective and causes the clouds to sink down a little into the atmosphere.
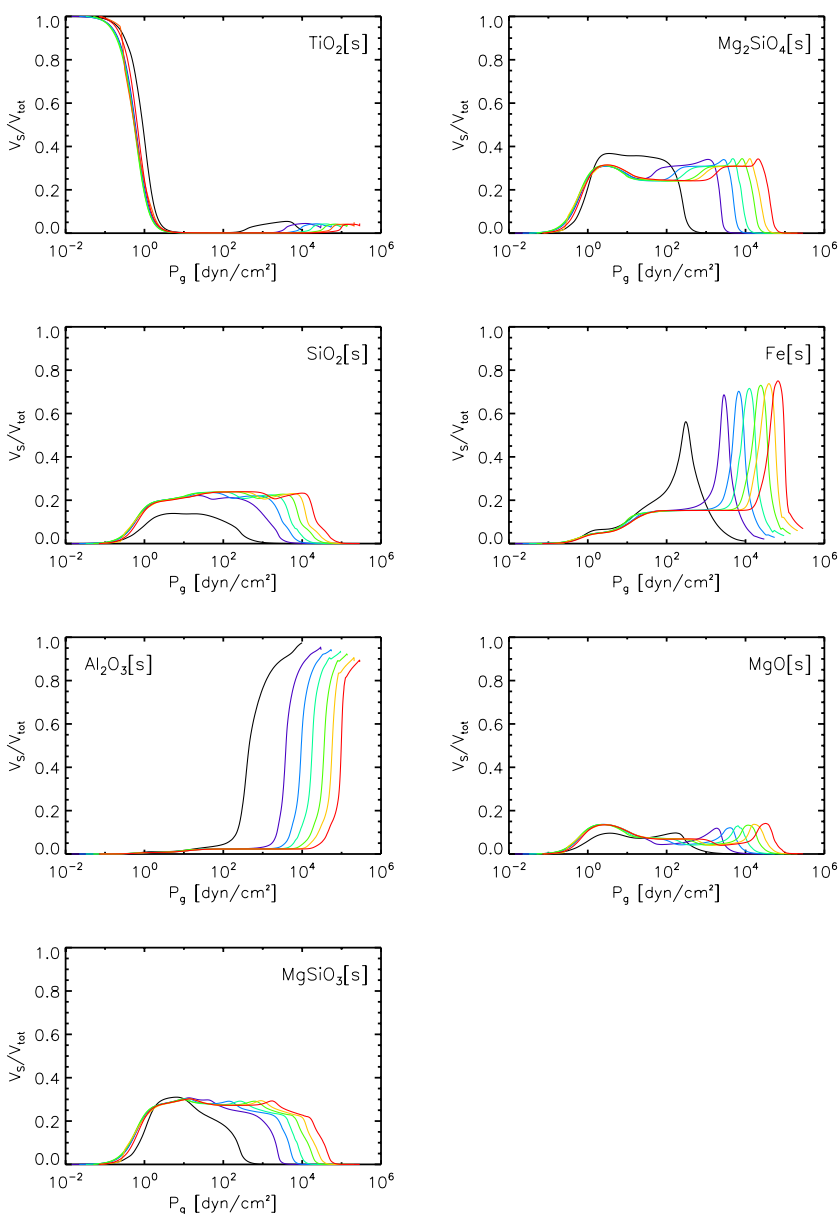

Fig. 4. Volume fraction of each of the seven solid species in a dust grain as a function of gas pressure. All fractions sum up to unity. The colorcoding is the same as in Fig. 3.

\subsection{Cloud particle details}

Figures 3 and 4 present a more detailed view of how the different processes involved in cloud formation depend on and react to each other as we move down through the atmosphere, as well as how the size and composition of the cloud particles changes in response.

Starting at the top of the atmosphere and moving down, the nucleation rate $J_{*}$ rises quickly due to increasing collisional rates as the density increases. When a distinct local temperature $T_{\theta} \approx$ $1300 \mathrm{~K}$ is exceeded, the nucleation rate drops to zero very fast. Consequently, the peak of the nucleation rate reaches deeper into the atmosphere the cooler the effective temperature of the model is. The nucleation rate causes a rise in the number density of dust grains $n_{\mathrm{d}}$, and the peak coincides with the first rapid increase in the number of dust particles.

After the nucleation rate peaks, the number density flattens out until it sharply increases again at the bottom of the cloud layers as the result of gravitational settling and cloud particle accumulation before complete evaporation. In the middle of the cloud layer, the cloud particle mass density $\rho_{\mathrm{d}}$ keeps increasing while the number density does not, showing that while the nucleation of new cloud particles has stopped, the already existing ones fall into deeper layers and are still growing larger. This coincides with the growing volume fraction of cloud particles other than $\mathrm{TiO}_{2}$ [s]. The silicates $\mathrm{Mg}_{2} \mathrm{SiO}_{4}[\mathrm{~s}], \mathrm{MgSiO}_{3}$ [s] and $\mathrm{SiO}_{2}[\mathrm{~s}]$ are the first to condense on the seed particles, quickly followed by $\mathrm{MgO}[\mathrm{s}]$ and $\mathrm{Fe}[\mathrm{s}]$ and then finally $\mathrm{Al}_{2} \mathrm{O}_{3}[\mathrm{~s}]$. 

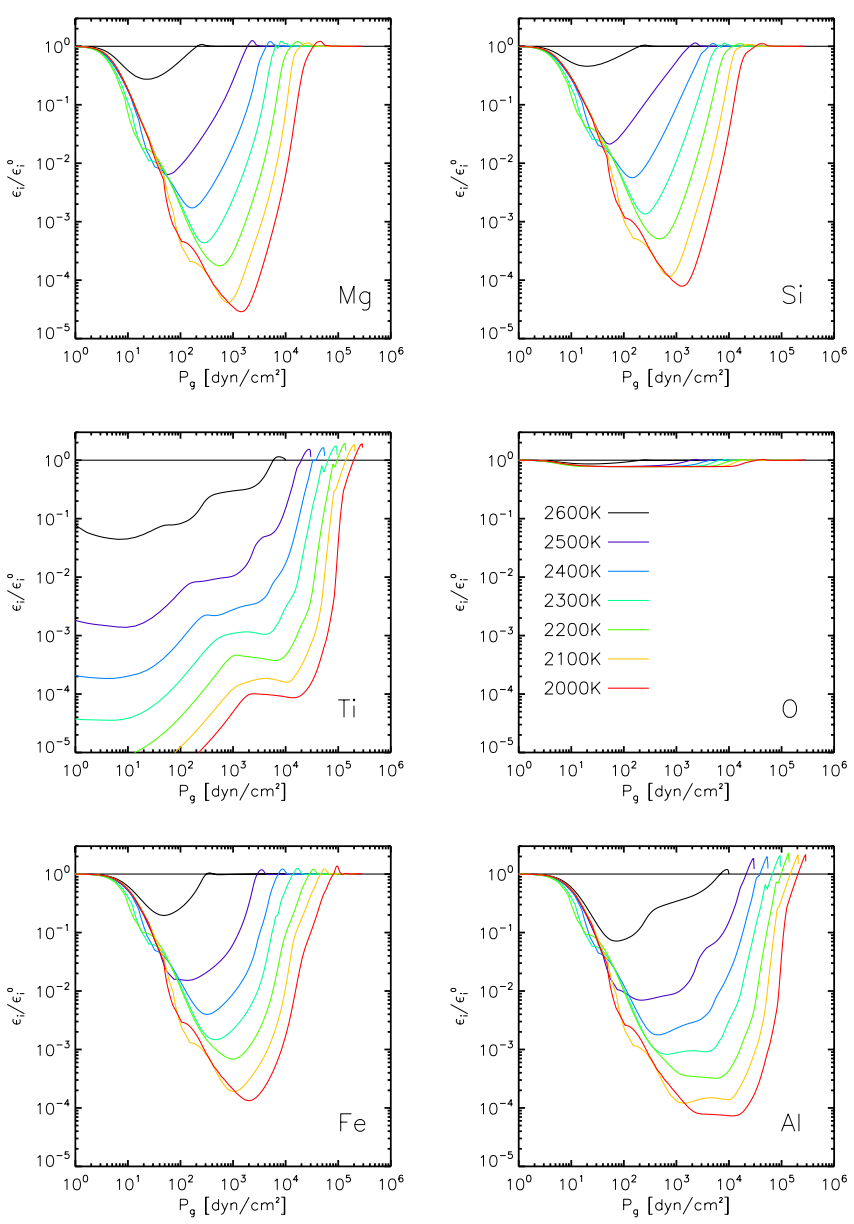

Fig. 5. Relative element depletion in the gas phase as a function of depth. Color-coding is the same as in Figs. 3 and 4 and is also indicated with the legend in panel 4.

At the bottom of the cloud layers the cloud particles evaporate at the high temperatures, causing a rapid decrease in their mass density and a drop in average particle size.

The net growth rate is $\chi_{\text {net }}>0$ when the grains are growing and $\chi_{\text {net }}<0$ when the grains are evaporating. The first growth period begins when we are far enough down in the atmosphere for the solids to effectively condense on the nucleation particles. It peaks before the nucleation rate indicating that it depends more on the amount of available surface area than on the formation of new small particles. The mean grain size $\langle a\rangle$ is determined by the net growth rate, and the first and second increase in the mean grain size happens in sync with the first and second period of growth. Near the bottom of the cloud layers the net growth rate and mean grain size rapidly drop as the cloud particles completely evaporate. The fluctuations in the net growth rate are due to the different solid species evaporating at different temperatures.

The drift velocity is initially decreasing as the gas density and therefore the friction - increases. The decreasing ends when the second period of growth sets in, as the larger cloud particles can more easily overcome the friction with the surrounding gas, because the downward accelerating force is proportional to grain size cubed (i.e., the grain mass), while the upward restoring force (the friction) is proportional to grain size squared.

\subsection{Element depletion}

The cloud particles are formed from the elements $\mathrm{Mg}, \mathrm{Si}, \mathrm{Ti}$, $\mathrm{O}, \mathrm{Fe}$ and $\mathrm{Al}$ in the present model. Figure 5 shows how their

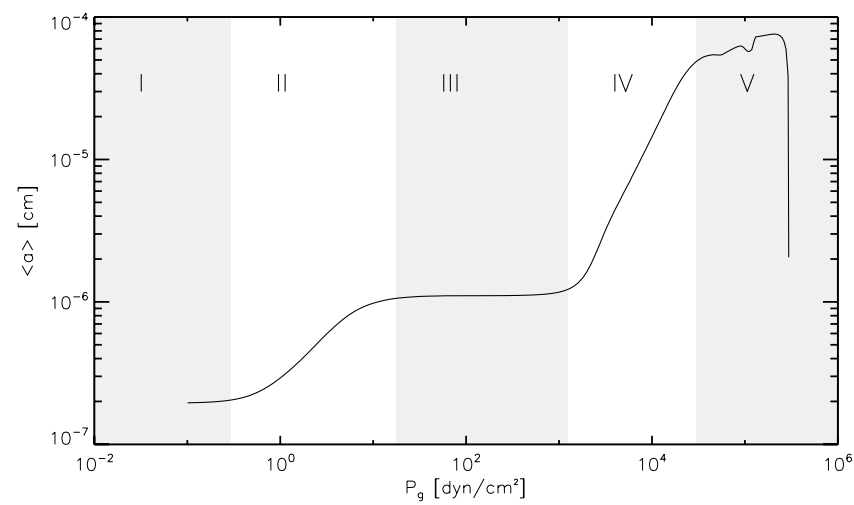

Fig. 6. The five regions of the clouds: I nucleation, II first growth, III drift, IV second growth, V evaporation.

abundances in the gas phase change as a function of atmospheric depth as they are bound in cloud particles. In general, the more rare elements are more strongly depleted. While the large abundance of $\mathrm{O}$ is barely affected by the cloud formation, the other elements are clearly depleted in the cloud-forming regions. Since we use $\mathrm{TiO}_{2}[\mathrm{~s}]$ as the seed particle, the relatively small abundance of $\mathrm{Ti}$ is strongly depleted in the upper layers. The element depletion of the remaining elements sets in a little later when there are available seed particles for them to condense on. The depletion is largest where the nucleation peaks (compare Figs. 5 and 3) and then decreases as the cloud particles reach the lower warmer layers and start to evaporate. Because the elements rain out with the cloud particles, we see an overabundance of condensable elements right below the cloud base, which is then transported upward with the gas convection. This will result in an increase of the corresponding gas opacity species.

\subsection{Cloud regions}

Based on the considerations in the previous section we can identify different regions within the clouds that each have their own characteristics and dominant processes. The formation, growth, and evaporation of the cloud particles in a gas of a given chemical composition is a function of temperature as well as gas pressure, and therefore to be understood as a "race" between the changing values of these two variables. Decreasing the temperature will enhance cloud particle formation, as will increasing gas pressure. The changing conditions for a cloud particle during its movement down through the photosphere, with its increasing temperature and increasing gas density, is therefore determined by the ratio of these two variables.

With respect to the grain size distribution, we can divide the clouds into five distinct zones based on how the mean size of a cloud particle changes as we move from the top to the base of the clouds. The five regions are illustrated for a model with $T_{\text {eff }}=2000 \mathrm{~K}, \log (g)=4.5$ and $[\mathrm{M} / \mathrm{H}]=0.0$ in Fig. 6. Similar to Woitke \& Helling (2004), they can be characterized as follows:

1. Nucleation. At the top of the cloud layers the nucleation of gas molecules is the dominant process, and the gas phase is therefore highly depleted in Ti relative to all other elements.

2. First growth. As the cloud particles fall down into the atmospheric layers, the increasing density and element replenishment allow for a growing number of possible surface reactions on the small seed particles, and the cloud particles increase considerably in size. As a result, the gas becomes more and more depleted in the elements that make up the 


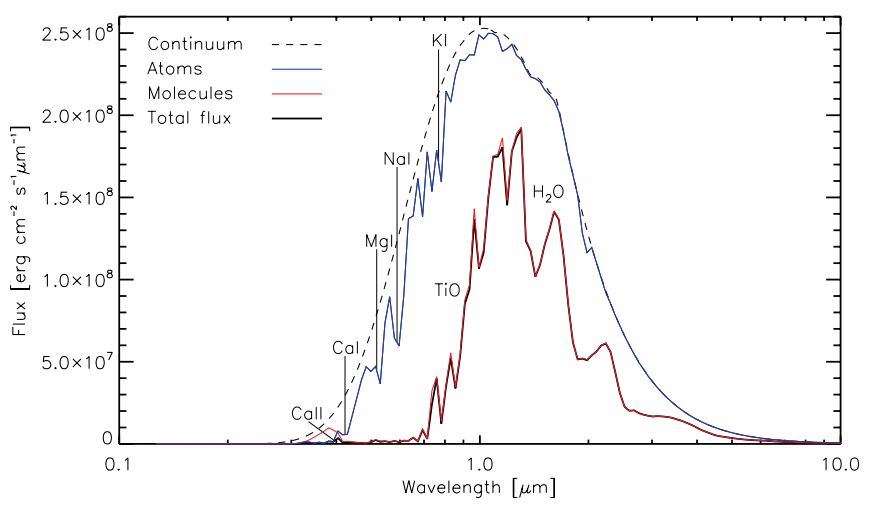

Fig. 7. Spectral contributions of gas opacity sources for a cloud-free MARCS-model atmosphere for $T_{\text {eff }}=2500 \mathrm{~K}, \log (g)=4.5,[\mathrm{M} / \mathrm{H}]=$ 0.0 .

cloud particles. The rate of newly forming seed particles still increases in this region, but it is the rapid growth that has the dominant effect on the average cloud particle size $\langle a\rangle$.

3. Drift. The increasing density of the gas combined with the increasing $\langle a\rangle$ causes the descent of the cloud particles to slow, which reduces the collision rate between the cloud particles and the gas molecules. This will decrease the growth rate. In the same region the nucleation rate peaks and the average cloud particle size remains constant, as the impeded growth of the large cloud particles is compensated for by the rapid formation of new small grains.

4. Second growth. When the nucleation rate suddenly drops, the growth in grain size is no longer balanced by the formation of small cloud particles, and the mean grain size therefore increases rapidly again. This ends the decrease of the drift velocity which remains more or less constant until the grains start evaporating. This and the still increasing density allow for an increase in the net growth rate.

5. Evaporation. In the lowest layers of the cloud particles start to evaporate as the temperature reaches the monomerization energies of the different solids, and the mean grain size decreases, dropping very fast as the last cloud particles evaporate at the cloud base.

We note that this stratification prevails as long as the hydrodynamic time scales are longer than any of the time scales representing cloud-formation processes.

\section{Synthetic spectra}

Detailed studies of the many complex physical and chemical processes that take place in our model atmospheres are an important part of understanding and developing our theories of stars, but at the end of the day it is only the light that leaves the atmosphere, the emitted spectrum, that provides us with a way to directly compare our stellar models with observations of real stars.

\subsection{Gas and cloud opacities}

Figure 7 illustrates the impact of atomic (blue) and molecular (red) line absorption on the spectrum of a dust free model with $T_{\text {eff }}=2500 \mathrm{~K}, \log (g)=4.5$ and $[\mathrm{M} / \mathrm{H}]=0$. At such low effective temperatures the absorption of atoms does not really affect the structure of the model, but they do create a few strong absorption lines in the ultraviolet and visible part of the spectrum. The most

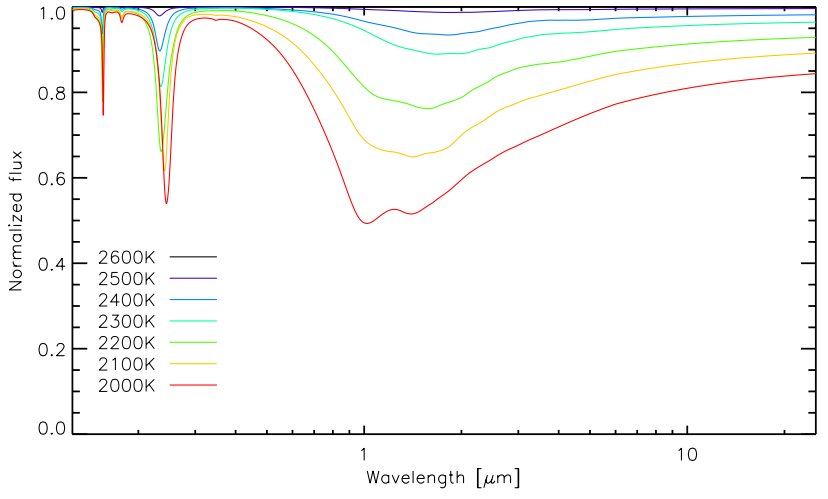

Fig. 8. Spectral contribution of dust (normalized with respect to the continuum flux) for models of decreasing effective temperature.

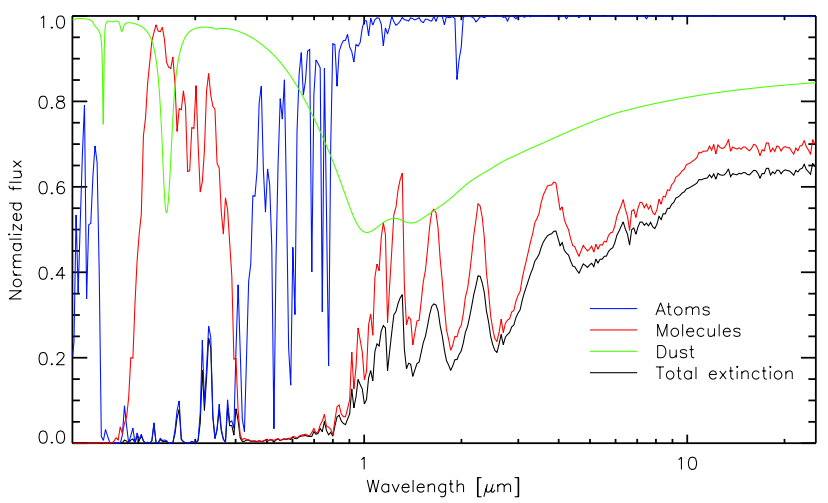

Fig. 9. Individual and combined effects of atomic opacity, molecular opacity and cloud opacity on the normalized flux (total flux divided by the continuum flux) of a cloud-forming DRIFT-MARCS-model atmosphere with $T_{\text {eff }}=2000 \mathrm{~K}, \log (g)=4.5$ and $[\mathrm{M} / \mathrm{H}]=0.0$.

prominent are the two CaII lines at $3968 / 3934 \AA$, the CaI line at $4227 \AA$, the MgI triplet at $5167 / 5173 / 5184 \AA$ the NaI doublet at $5890 / 5895 \AA$, and the KI doublet at 7665/7699 $\AA$ (Walker 2014). Still, it is the molecules that dominate the spectrum, completely obscuring most of the atomic lines except in the ultraviolet region. A more detailed look at the individual absorption of the molecules is presented in Appendix B.

Figure 8 shows how the increasing dust opacity affects the spectrum for models of decreasing effective temperatures. The normalized flux (the flux divided by the continuum flux) includes only the effect of dust on the spectrum. The dust opacity increases in a broad band that covers the optical and near-infrared wavelength regions, peaking at around $1-3 \mu \mathrm{m}$. For our coolest model, about half of the light is being blocked by the cloud layers at $\lambda \approx 1 \mu \mathrm{m}$. This is similar to the effect of water vapor in our cloud-free $T_{\text {eff }}=2500 \mathrm{~K}$ model (Fig. 7), and to (water) clouds in Earth's atmosphere.

In Fig. 9 we plot the effect of dust opacity (green) on the spectrum as a function of wavelength in comparison to the various gas opacity contributions (atoms - blue, molecules - red) for a cloud-forming DRIFT-MARCS-model atmosphere $\left(T_{\text {eff }}=\right.$ $2000 \mathrm{~K}, \log (g)=4.5$ and $[\mathrm{M} / \mathrm{H}]=0.0)$. The whole wavelength range of MARCS is shown. The dust extinction is most prominent in a broad band that covers the optical and near-infrared regions and peaks at around $\lambda=1-2 \mu \mathrm{m}$, where it is comparable to or even greater than the molecular absorption. For $\lambda>10 \mu \mathrm{m}$ the dust extinction has a noticeable dampening 

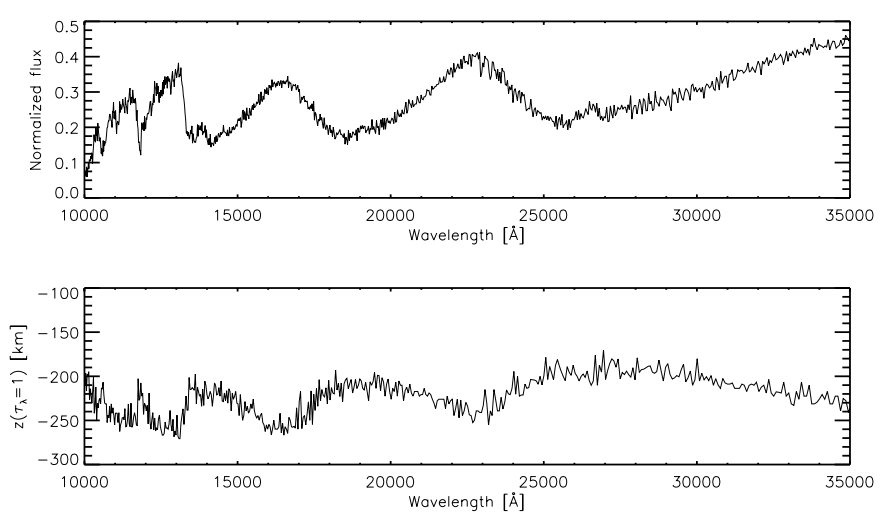

Fig. 10. Total normalized flux (top) and the atmospheric depth, $z\left(\tau_{\lambda}=1\right)$ for cloud-forming DRIFT-MARCS-model atmosphere for $T_{\text {eff }}=2000 \mathrm{~K}, \log (g)=4.5,[\mathrm{M} / \mathrm{H}]=0.0$. The total geometric extension of the atmosphere from $\log \left(\tau_{\text {ROSS }}\right)=2$ to $\log \left(\tau_{\text {ROSS }}\right)=-10$ is $333 \mathrm{~km}$.

effect on the molecular absorption bands, which would otherwise have completely dominated the spectrum. The two sharp peaks at short wavelengths are numerical artifacts pointing to challenges with the Mie calculations. They, however, occur in the ultraviolet part of the spectrum where the opacity is heavily dominated by atomic absorption and therefore do not have an effect on the spectrum (nor on the structure of the model). The dust extinction increases with decreasing effective temperatures.

\subsection{Optical depth}

The opacity of the atmosphere changes with wavelength and therefore so does the optical depth $\tau(\lambda)$. We can determine the optical depth of the atmosphere from our synthetic spectrum and thereby estimate how deep into the atmosphere we can see at a specific wavelength. In Fig. 10 we plot the total normalized flux (top) and the geometrical depth, $z(\lambda)$, where $\tau(\lambda)=1$ in a cloud-forming atmosphere with $T_{\text {eff }}=2000 \mathrm{~K}, \log (g)=4.5$ and $[\mathrm{M} / \mathrm{H}]=0.0$. When the opacity is high, the flux is low and we cannot see as far into the atmosphere as when the opacity is low and the flux is high. Figure 10 demonstrates that the overall flux in the near infrared is absorbed by $>50 \%$. An additional flux variation of $\approx 10 \%$ translates into a $\Delta z(\lambda) \approx 50 \mathrm{~km}$ which is $15 \%$ of the total geometrical extension of the atmosphere of a $\log (g)=4.5$-type ultra-cool object as seen in Fig. 10. This is the cause of the observable variation in an exoplanet transit depth as a function of wavelength and is the direct cause that transit observations can be translated into exoplanetary spectra and structure.

\subsection{Comparison to observed spectra}

Ultra-cool dwarfs emit the majority of their radiation flux at near-infrared (NIR) wavelengths and their discovery and classification is therefore mainly conducted by NIR spectroscopic instruments. One such instrument is the SpeX spectrograph mounted on the 3 m NASA Infrared Telescope Facility, which provides moderate- and low-resolution broad-band NIR spectra (Rayner et al. 2003). SpeX spectra has been proven ideal for NIR classification, characterization of atmospheric and physical properties, as well as testing atmosphere models (Burgasser 2014), and is made readily available from the online SpeX Prism
Spectral Libraries ${ }^{1}$ that we have compared with our synthetic DRIFT-MARCS spectra.

The SpeX spectra are all normalized, have a resolution of $R=\lambda / \Delta \lambda \approx 120$, and span a wavelength range of $\lambda \approx$ $0.65-2.55 \mu \mathrm{m}$. For a single comparison, we re-sampled our synthetic spectrum to match the resolution and range of the observed spectrum and then fitted the synthetic spectrum to the observed spectrum by simply scaling the synthetic spectrum. We used the non-linear least squares curve-fitting routine MPFIT (Markwardt 2009) which identifies the best fit as the one with the lowest value of

$\chi^{2}=\left(\sum_{i=1}^{N} \frac{\left(A \cdot f_{\text {synth }, \mathrm{i}}-f_{\mathrm{obs}, \mathrm{i}}\right)^{2}}{\sigma_{\mathrm{obs}, \mathrm{i}}}\right) /(N-1)$,

where $A$ is the scaling factor, the only free parameter, and $N$ is the number of data points. We repeated this process for every combination of synthetic and observed spectrum, in the end identifying the best fitting synthetic spectrum for each observed spectrum as the one with the lowest value of $\chi^{2}$.

Most of the $\chi^{2}$ values were in the range of $\chi^{2} \approx 1.5-15$ with a few very large exceptions. Since our grid serves as an indication of the direction we are going in with our models, we expect that a good deal of the fits will be considerably improved once we have computed a more complete grid that includes variations in surface gravity or metallicity. We are therefore wary of systematic offsets in our fits and only consider the best fits of a few selected spectral sub classes, where $\chi^{2}<2.5$ is low enough to assume a true match between synthetic and observed spectra.

Representative stars within the parameters of our small grid for the present project and their best fit models are presented in Table 5 and Figs. 11 and 12. These objects have not been presented in Witte et al. (2011). Here, we focus on mid- to late-type M-dwarfs (Sect. 4.3.1), early- to mid-type L-dwarfs (Sect. 4.3.2), and on warm, giant gas planets (Sect. 4.3.3). We specifically address the giant gas planet WASP19b as one example.

\subsubsection{Mid- to late-type $M$ dwarfs}

In Fig. 11 we present the comparison between the observed spectra of six M-dwarfs and our best fit models. The earliest subtype that can be fitted by our models is M4.5. With an effective temperature of $T_{\text {eff }}=3000 \mathrm{~K}$ its atmosphere is dust free, and its spectrum is generally well modeled by the synthetic spectrum of our model. The famous TiO bands of M-dwarfs dominate the total absorption from $0.7-1.0 \mu \mathrm{m}$, only disturbed slightly by $\mathrm{VO}$ at $0.8 \mu \mathrm{m}$ and $\mathrm{CrH}$ at $0.85-0.9 \mu \mathrm{m}$. The absorption band in the model at $\lambda=1.0 \mu \mathrm{m}$ is a mix of $\mathrm{CrH}$, TiO, and $\mathrm{FeH}$ in order of influence, but it is not observed in the spectra of this star. For $\lambda>1.3 \mu \mathrm{m}$ the broad absorption bands of $\mathrm{H}_{2} \mathrm{O}$ become the main absorption features and they stay almost undisturbed by other molecules and atoms except at $\lambda=2.3-2.4 \mu \mathrm{m}$ where $\mathrm{CO}$ absorption causes the small fluctuations. The absorption is somewhat underestimated at $\lambda=1.4-1.7 \mu \mathrm{m}$ for most of the models and slightly overestimated at $\lambda=1.8-2.3 \mu \mathrm{m}$. We note however that the deviation is not correlated with $T_{\text {eff }}$, and that the magnitude of the two deviations are not correlated with one another. We therefore conclude that the mismatch is most likely due to chemical abundance effects beyond the range of our present grid.

None of the M-dwarfs reach effective temperatures below $T_{\text {eff }}=2600 \mathrm{~K}$, and if any cloud formation takes place in their atmosphere, it plays no significant role in their spectra. As their

1 http://wWW . browndwarfs .org/spexprism 
D. Juncher et al.: Atmosphere modeling with cloud formation

Table 5. Name, spectral type, and data reference for the observed spectra together with the parameters of our best fit model.

\begin{tabular}{llr|rc}
\hline \hline Object & & & \multicolumn{2}{|c}{ Best fit model } \\
name & SpT & Reference & $T_{\text {eff }}$ & $\chi^{2}$ \\
\hline 2MASS J12471472-0525130 & M4.5 & Kirkpatrick et al. (2010) & $3000 \mathrm{~K}$ & 1.41 \\
Gliese 866AB & M5.6 & Burgasser et al. (2008) & $2900 \mathrm{~K}$ & 1.60 \\
2MASS J11150577+2520467 & M6.5 & Burgasser et al. (2004) & $2800 \mathrm{~K}$ & 2.02 \\
VB 8 & M7 & Burgasser et al. (2008) & $2800 \mathrm{~K}$ & 1.77 \\
2MASS J17364839+0220426 & M8 & Burgasser et al. (2004) & $2700 \mathrm{~K}$ & 2.05 \\
2MASS J11240487+380854 & M8.5 & Burgasser et al. (2004) & $2600 \mathrm{~K}$ & 2.49 \\
2MASSW J0320284-044636 & M8/L0.5 & Burgasser et al. (2008) & $2500 \mathrm{~K}$ & 2.22 \\
2MASS J15500845+1455180 & L2 & Burgasser et al. (2009) & $2000 \mathrm{~K}$ & 2.33 \\
2MASSW J0036159+182110 & L3.5 & Burgasser et al. (2008) & $2100 \mathrm{~K}$ & 2.09 \\
2MASS J1104012+195921 & L4 & Burgasser et al. (2004) & $2000 \mathrm{~K}$ & 2.08 \\
SDSS J154849.02+172235.4 & L5 & Chiu et al. (2006) & $2000 \mathrm{~K}$ & 1.91 \\
2MASS J14162409+1348267 & L6 & Schmidt et al. (2010) & $2300 \mathrm{~K}$ & 2.20 \\
\hline
\end{tabular}

Notes. All models have $\log (g)=4.5$ and $[\mathrm{M} / \mathrm{H}]=0$.
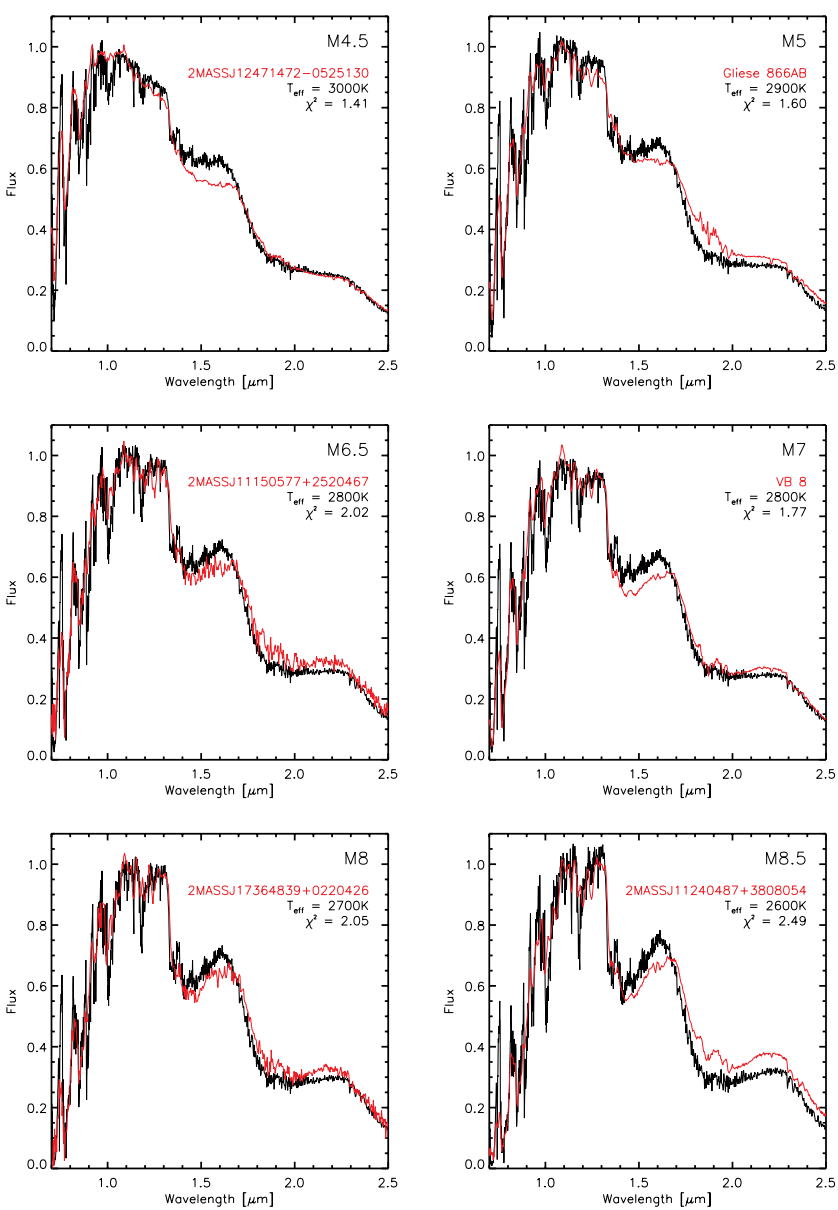

Fig. 11. Mid- and late M-dwarf SpeX observations fitted with DRIFT-MARCS.

effective temperatures decrease, the peak of their spectrum shifts towards longer wavelengths. The intensity of the $\mathrm{TiO}$ bands grows larger and the bands are blended with the increasing absorption of $\mathrm{VO}$ and $\mathrm{CrH}$. The absorption of $\mathrm{CaH}$ also increases at $\lambda=0.7-0.75 \mu \mathrm{m}$ but has a very small effect on the spectrum. The increase in absorption of $\mathrm{CrH}, \mathrm{VO}$ and $\mathrm{FeH}$ at $\lambda=1.0 \mu \mathrm{m}$ is well matched by the models. Finally, the absorption of $\mathrm{H}_{2} \mathrm{O}$ in the infrared increases significantly as well, each band growing deeper with decreasing effective temperature. With the massive
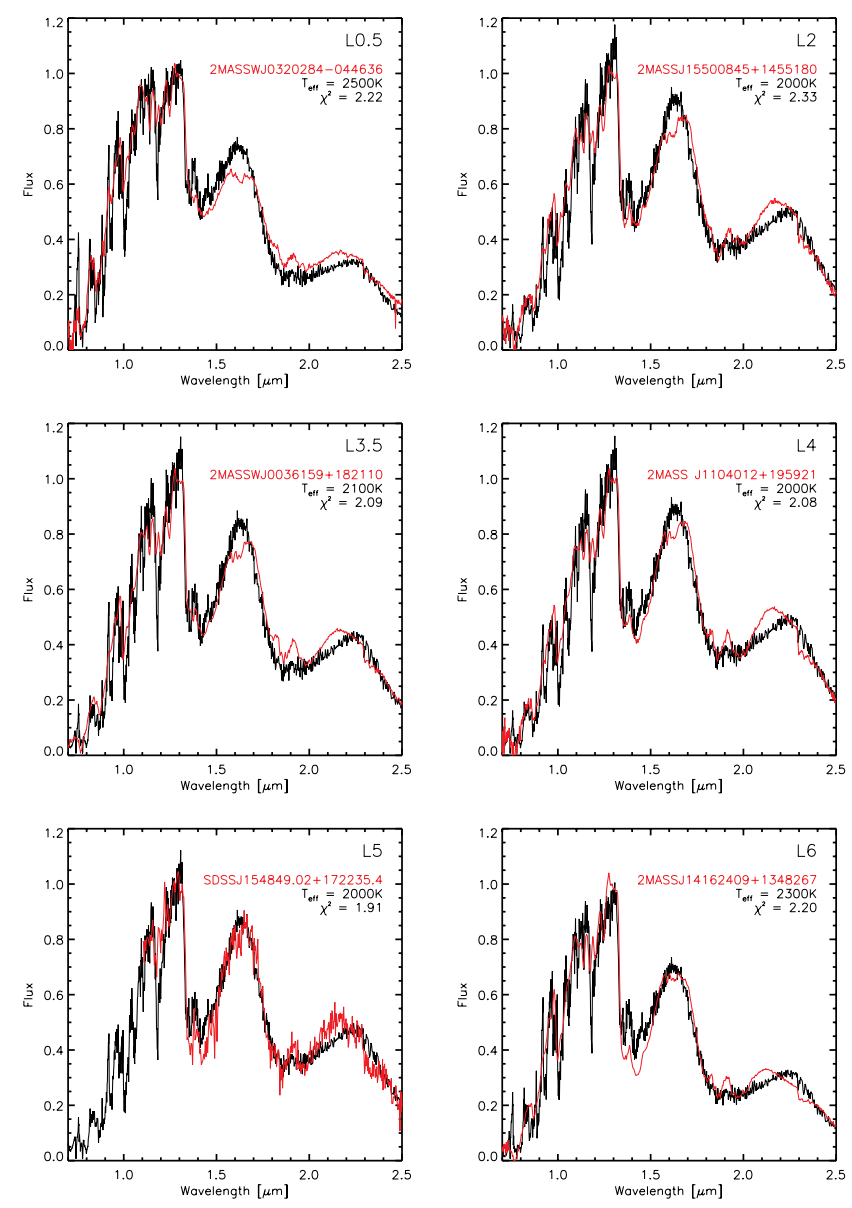

Fig. 12. Early- and late L-dwarf SpeX observations fitted with DRIFT-MARCS.

suppression of the continuum due to the absorption of molecules, the coolest M-dwarfs are clearly far away from being ideal black body radiators. As demonstrated in Fig. 2, clouds barely affect the atmosphere of objects with $T_{\text {eff }}>2600 \mathrm{~K}$.

\subsubsection{Early- and mid-type L-dwarfs}

In Fig. 12 we present the comparison between the observed spectra of six L-dwarfs and our best fit models. The latest sub-type 
that can successfully be fitted by the model grid in our present work is L6. For later sub-types $\chi^{2}$ becomes too large as the effective temperatures drop below our lowest grid temperature of $T_{\text {eff }}=2000 \mathrm{~K}$. For decreasing effective temperatures, the absorption from $0.7-1.0 \mu \mathrm{m}$ gradually becomes characterized by equally strong $\mathrm{TiO}$ and $\mathrm{VO}$ bands. The absorption of $\mathrm{CaH}$ and $\mathrm{CrH}$ also becomes stronger in that region, but since their bands tend to coincide with the stronger $\mathrm{TiO}$ and $\mathrm{VO}$ bands, they do not affect the spectrum that much. The absorption feature at $\lambda=1.0 \mu \mathrm{m}$ is the result of a peak in $\mathrm{CrH}$ absorption as well as absorption from $\mathrm{TiO}$ and $\mathrm{FeH}$. The other noticeable absorption features at $\lambda=1.2 \mu \mathrm{m}$ are caused by the superposition of the absorption peaks of $\mathrm{CrH}, \mathrm{H}_{2} \mathrm{O}, \mathrm{VO}$ and $\mathrm{CaH}$ and $\mathrm{FeH}$.

The infrared part of the SpecX spectral region is dominated by three strong absorption features at $1.4 \mu \mathrm{m}, 1.9 \mu \mathrm{m}$ and $2.5 \mu \mathrm{m}$, and corresponding opacity minima at $1.6 \mu \mathrm{m}$ and $2.2 \mu \mathrm{m}$. The intensity of the absorption bands are determined by a temperature-, pressure- and elemental abundance-dependent combination of $\mathrm{CO}$ and $\mathrm{H}_{2} \mathrm{O}$ and could also include contributions by other species with yet incomplete opacity data. The flux at the intensity minima are to a large extent determined by the more continuumlike dust absorption. The slight mismatch between our synthetic spectra and the SpecX infrared observations could therefore be due to incomplete inclusion of a combination of any of these factors, but it is probably more likely (since there is no clear $T_{\text {eff }}$ dependence on the quality of the fits) to be due to the smallness of the grid of models yet, which does not allow us to vary the chemical abundances and gravity sufficiently for more accurate fits to the observed spectra. The present paper is, however, not aimed at determining the parameters of the presented objects by detailed spectral fitting, but rather at developing the basic principles of incorporating self-consistent dust formation into the gas phase atmospheric computation, and test whether such models relate realistically to observations. As such, Figs. 11 and 12 fully serve their purpose of demonstrating that this has been achieved, and we will leave the detailed matching to a future paper with a more extended grid.

In general we see that the optical region is dominated by $\mathrm{TiO}$ and $\mathrm{VO}$ absorption bands, the near-infrared region by strong metal hydrid bands $(\mathrm{CrH}, \mathrm{FeH}$, and $\mathrm{CaH})$, and the infrared region by the broad, cloud-opacity-dampened $\mathrm{H}_{2} \mathrm{O}$ absorption bands.

We note that our best fit model to 2MASS J1416 is several hundred Kelvin warmer than a typical L6-type dwarf. This particular L dwarf has been identified as an unusually blue object for its spectral type (Bowler et al. 2010), and it is therefore likely that non-solar metallicities or other effects make it impossible for our small grid of models to fit it correctly.

Furthermore, 2MASSW J0320 might be an unresolved late $\mathrm{M}+\mathrm{T}$ dwarf binary system (Burgasser et al. 2008) and can in that case not be fitted well by a single model spectrum.

\subsubsection{WASP-19b}

Hot Jupiters have deep hydrogen-helium atmospheres. Some of them orbit so close to their parent stars that they have surface temperatures larger than $T=2000 \mathrm{~K}$. We apply DRIFT-MARCS to model such an atmosphere where we do not yet take into account the irradiation by the host star.

The atmosphere of WASP-19b was modeled by Anderson et al. (2013) using the spectral retrieval methods developed in Madhusudhan \& Seager (2009, 2010, 2011), which utilize parametric $\left(T_{\text {gas }}, P_{\text {gas }}\right)$ structures in combination with a cloud-free gas made of $\mathrm{H}_{2}, \mathrm{H}_{2} \mathrm{O}, \mathrm{CO}, \mathrm{CH}_{4}, \mathrm{CO}_{2}$, and $\mathrm{NH}_{3}$. In the retrieval approach, the $\left(T_{\text {gas }}, P_{\text {gas }}\right)$ structure and the
Table 6. Relative flux of the exoplanet WASP-19b with respect to its star at different wavelengths (Table 4, Anderson et al. 2013).

\begin{tabular}{lll}
\hline \hline Wavelength & $F_{\mathrm{p}} / F_{\star}$ & Reference \\
\hline $1.6 \mu \mathrm{m}$ & $0.00276 \pm 0.00044$ & Anderson et al. (2010) \\
$2.09 \mu \mathrm{m}$ & $0.00366 \pm 0.00067$ & Gibson et al. (2010) \\
$3.6 \mu \mathrm{m}$ & $0.00483 \pm 0.00025$ & Anderson et al. (2013) \\
$4.5 \mu \mathrm{m}$ & $0.00572 \pm 0.00030$ & Anderson et al. (2013) \\
$5.8 \mu \mathrm{m}$ & $0.0065 \pm 0.0011$ & Anderson et al. (2013) \\
$8.0 \mu \mathrm{m}$ & $0.0073 \pm 0.0012$ & Anderson et al. (2013) \\
\hline
\end{tabular}

molecular abundances are fitting parameters used to retrieve the observed spectrum. In the DRIFT-MARCs approach, on the other hand, the $\left(T_{\text {gas }}, P_{\text {gas }}\right)$ structure and the abundances of the individual gas- and dust-species are computed from first principles self-consistently with the radiative transfer, energy balance, opacities, and dust formation, as described above. There are therefore no free parameters in DRIFT-MARCS spectrum simulations (but, as mentioned above, irradiation is not yet included in the version presented here), and the best fit model gives direct information about the temperature profile and the chemical composition of the planetary atmosphere.

WASP-19b is a transiting exoplanet with a mass of $M_{\mathrm{p}}=$ $1.165 M_{\mathrm{J}}$ and a radius of $R_{\mathrm{p}}=1.383 R_{\mathrm{J}}$ in a close orbit around its parent star with a period of only $P=0.789$ days, as determined from transit and radial velocity measurements. It is therefore classified as a hot Jupiter. The day-side flux of WASP-19b has been measured by observing the occultation of the planet by its parent star with the Spitzer Space Telescope. The relative flux of the planet with respect to its star is presented in Table 6 (Table 4, Anderson et al. 2013).

The parent star of WASP-19b is a G8V-type star given by Anderson et al. (2013) as $T_{\text {eff }}=5475 \mathrm{~K}, \log (g)=4.43$ and $[\mathrm{M} / \mathrm{H}]=0.02$. We used DRIFT-MARCS to compute a stellar model atmosphere with these parameters and calculated its synthetic spectrum, the flux $f_{\star}$. The relative flux $F_{\mathrm{p}} / F_{\star}$ which we receive on the Earth from the two is

$\frac{F_{\mathrm{p}}}{F_{\star}}=\frac{f_{\mathrm{p}}}{f_{\star}}\left(\frac{R_{\mathrm{p}}}{R_{\star}}\right)^{2}$,

where $\left(R_{\mathrm{p}} / R_{\star}\right)^{2}=0.02050 \pm 0.00024$ is the planet-to-star area ratio. We calculated $F_{\mathrm{p}} / F_{\star}$ for each of our cloud-forming DRIFT-MARCs models, setting $f_{\mathrm{p}}$ as their respective fluxes. Comparing these synthetic planet-to-star fluxes with the observed planet-to-star flux, we found that it is best described by our cloud-forming, non-irradiated model with $T_{\text {eff }}=2600 \mathrm{~K}$, $\log (g)=3.2$ and $[\mathrm{M} / \mathrm{H}]=0$.

The spectrum is quite insensitive to the value of $\log (g)$, which therefore cannot be determined from the spectrum $(\log (g)=3.2$ and 4.5 gives basically identical IR spectra at this resolution as is demonstrated in Fig. 13). Instead we adopt the value $\log (g)=3.2$ from determination of the planetary mass and radius, as given in Anderson et al. (2013). Our preliminary grid presented here is only calculated for solar metallicity (in agreement with what is found for WASP-19, but WASP-19b could obviously be metallicity enhanced). Our model fit is therefore nothing other than a rough first temperature fit and, for the purpose of the present paper, mainly a working demonstration that our model method is able to self-consistently reproduce even hot exoplanetary spectra. There are no other self-consistent temperature estimates in the literature for us to use as comparison, but Anderson et al. (2013) attempted an estimate of the planetary 


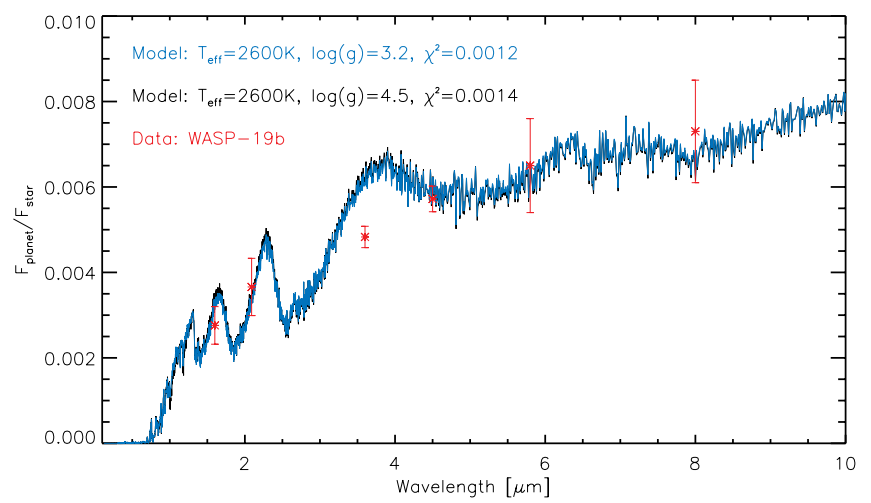

Fig. 13. Best-fit synthetic transit spectrum for WASP-19b for $\log (g)=$ 4.5 (blue) and $\log (g)=3.2$ (green), based on DRIFT-MARCs model atmosphere simulations for the $\operatorname{star}\left(T_{\text {eff }}=5475 \mathrm{~K}, \log (g)=4.43\right.$, $[\mathrm{Fe} / \mathrm{H}]=0.02)$ and for the planet. The Spitzer data points are plotted in red. We derive that the temperature of the planet is $T_{\mathrm{eff}}=2600 \mathrm{~K}$

effective temperature by calculating a brightness temperature obtained by dividing a Planck function for various guesses of the effective temperature of the planet with a Planck function of the stellar effective temperature, and fitting this ratio to the Spitzer measurements. In this way they found values between $2260 \mathrm{~K}$ and $2750 \mathrm{~K}$ (depending on the filter they fitted to). They also estimated the planetary equilibrium temperature based on the known effective stellar temperature and the planetary orbital size, and by assuming a planetary albedo of zero. In this way they reached effective temperatures of WASP-19b between $2040 \mathrm{~K}$ and $2614 \mathrm{~K}$ (or actually, between $2433 \mathrm{~K}$ and $3109 \mathrm{~K}$ when correcting for a missing $\sqrt{2}$ in their formula for calculating $T_{\text {eff }}$ as given in the caption to their Table 3 ). The range in temperature reflects a range in assumed efficiency in energy transport from day- to night-side of the planet. They also investigated whether or not a temperature inversion was visible in the measured flux distribution, and concluded that their temperature inversion profile was inconsistent with the observed flux distribution (however, with it seemingly lacking the spectral features of the inversion in their computed spectra, due to lacking chemical equilibrium and relevant opacities in the computations). Our estimate of $T_{\text {eff }}=2600 \mathrm{~K}$ is in good agreement with the estimate by Anderson et al. of an atmosphere with solar $\mathrm{C} / \mathrm{O}$ ratio, albedo zero and no temperature inversion. This is encouraging because it qualitatively indicates an atmosphere with low albedo, that is relatively clear, absorbs most of the incoming energy in the bottom of the atmosphere (with winds that will transport energy to the back side, but not so efficient that the planet reaches equal day and night temperature), and has no sign of a strong temperature inversion (with the caution that neither we nor Anderson et al have effectively analyzed the effect of a temperature inversion, due to the two different computational limitations mentioned above). Figure 13 shows the comparison of our synthetic spectrum and the Spitzer observations.

\section{Cloud particle porosity}

Material properties are an essential input for every model. The challenge of obtaining such input has recently been outlined in Fortney et al. (2016). Here we shortly discuss the effect of porosity on the cloud opacity.

Figure 14 shows that the porosity of cloud particles can have a considerable effect on the opacity. We chose to represent the

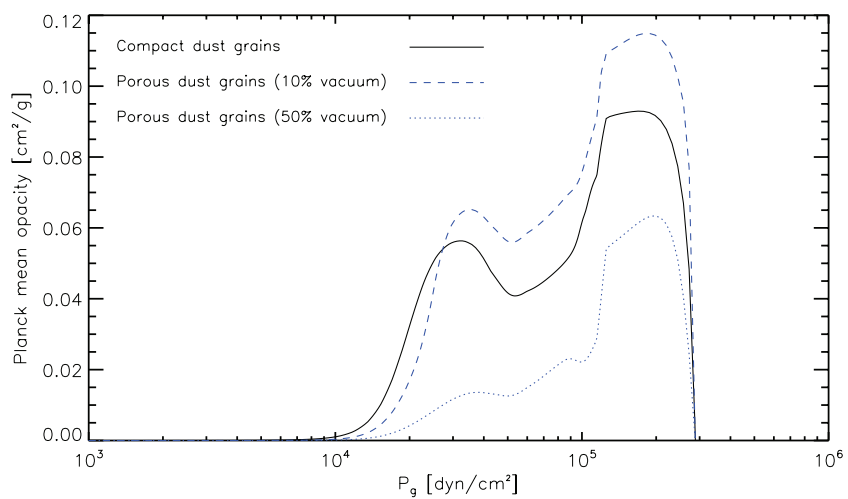

Fig. 14. Effect of porosity on the cloud opacity $\left(T_{\text {eff }}=2000 \mathrm{~K}, \log (g)=\right.$ 4.5 and $[\mathrm{M} / \mathrm{H}]=0.0)$. The plotted cloud opacity is the Planck mean opacity.

effect in terms of local Planck mean opacities as this allows us to plot a meaningful measure of the opacity as a function of the whole atmospheric extension. We compare the integrated opacity for one example cloud-forming model atmosphere $\left(T_{\mathrm{eff}}=\right.$ $2000 \mathrm{~K}, \log (g)=4.5,[\mathrm{M} / \mathrm{H}]=0.0)$ for three types of cloud particles: one is compact (using the results directly from Figs. 3 and 4; solid line), one contains $10 \%$ vacuum (dashed line), and one contains $50 \%$ vacuum (dotted line). We test this by adding the vacuum as an eighth condensate and then scaling the contribution of each condensate such that the total volume of a single dust grain - and thereby also its surface area - remains the same, while its mass decreases for increasing porosity. The model atmosphere has not been iterated with the new cloud opacity; we have simply re-calculated the opacity of the cloud layer in the already converged model to illustrate if there is an effect. Interestingly, we see that by increasing the porosity slightly the cloud grains become more opaque. If the porosity is too high, the opacity drops again since the light can pass unhindered through a large part of the cloud grains.

Porosity could arise if the cloud particles do not attain a compact shape during their formation or evolution, but rather develop fractal shapes instead. We are familiar with this process from Earth's atmosphere as "snow". Comets are examples of a different kind of porosity. It is not clear whether potential porosity can be sustained as the cloud particles fall into deeper atmospheric layers where their frictional interaction with the gas increases, which then would lead to a compactification or break-off of dangling structures. A more realistic scenario for relatively hot atmospheres could be that different materials evaporate at different temperatures, while others remain thermally stable throughout the entire atmosphere.

\section{Conclusions}

The coming years and decades will see a substantial technological development that will make it possible to obtain direct spectra of increasing quality of nearby exoplanets. Reliable interpretation of such high-quality spectra will require detailed complex self-consistent model atmospheres, which at the same time will make it possible to reliably quantify such exciting features as potential biomarkers in the atmosphere, and hence open a route for the first scientific discussions of possible life forms on nearby extrasolar planets. With these long-term goals in mind we have taken the first steps to combine two well-tested computer codes from stellar atmospheric theory, namely the 
MARCS radiative and convective equilibrium code for gaseous atmospheres and the DRIFT dust and cloud-formation code. In combination, the DRIFT-MARCS code that we here have presented for the first time, is able to compute self-consistent model atmospheres that can include both radiative-convective energy transport, the chemical equilibrium between both gas and dust species, as well as cloud formation and cloud destruction. These are necessary ingredients to compute self-consistent models of exoplanetary atmospheres, and the exercise serves a double purpose, namely to pave the way for DRIFT-MARCS self-consistent general exoplanetary models and to increase the accuracy of the stellar models of M-, L- and T-type stars (and brown dwarfs), whose orbiting exoplanets we already today are able to obtain crude spectra of.

M-, L-, and T-dwarfs are very attractive targets when searching for new exoplanets by indirect methods. Their relatively small mass and size provide stronger signals for detection with the radial velocity, astrometry, and transit methods. An inherent problem of these exoplanet search methods is that the uncertainty of the properties of the host star propagates to the properties of its planet. It is therefore crucial that the stellar models linking the observations of a star to its properties are as precise as possible, and the ultra-cool dwarf stars are much more complex to model than their larger and hotter cousins, mainly because their temperatures are low enough for mineral clouds to form in their atmospheres. We have demonstrated when and how the mineral cloud formation starts to play a role for the atmospheric structure of our models, and we have shown that emergent spectra based on our DRIFT-MARCS model atmospheres are in good agreement with observed spectra for the whole range of spectral types from mid-type M-dwarfs to late-type L-dwarfs $\left(T_{\text {eff }}=3000 \mathrm{~K}\right.$ to $\left.2000 \mathrm{~K}\right)$. The DRIFT-MARCS code is therefore already in its present form a reliable tool to accurately determine the stellar parameters and hence improve the parameters of exoplanets orbiting cool dwarf stars.

Hot Jupiter exoplanets orbiting solar-type and warmer stars are themselves of comparable $\left(T_{\text {eff }}, \log (g)\right)$ values to the ultracool dwarf stars, and one would expect them to have slow or tidally locked rotations. They will therefore to a large extent resemble the ultra-cool dwarf stars, and can therefore to a first approximation be modeled in the same way as these. Crude spectra or photometric data points can already today be obtained for a few hot Jupiter exoplanets by subtracting the stellar spectrum during occultation from the stellar spectrum with the exoplanet in different phases (i.e., positions of its orbit). We therefore tested our computed DRIFT-MARCS synthetic spectra against photometric data of the hot Jupiter WASP-19b obtained from the Spitzer satellite. We found good agreement between the observed photometry and a spectrum based on a DRIFT-MARCS model with $T_{\text {eff }}=2600 \mathrm{~K}$.

Gas giants in larger orbits will show more complicated structures due to their more normal rotation speed, and will require more dynamic features and more extensive chemical gas and dust calculations included in the modeling due to their lower temperature. This will be the subject of forthcoming papers and more advanced versions of the DRIFT-MARCS code than presented here, as will the modeling of even more Earth-like exoplanets.

Acknowledgements. We are thankful to the University of St Andrews for hospitality and financial support toward D.J. during an extended stay in 2014 as part of her Ph.D. Thesis work, where part of this work was done. Ch.H. highlights financial support from the European Community under the FP7 by the ERC starting grant 257431 . We greatly appreciate discussions with, and valuable comments from, B. Gustafsson and K. Lodders, and are thankful for an inspiring and thorough referee report from $\mathrm{D}$. Homeier. This research has benefitted from the SpeX Prism Spectral Libraries, maintained by Adam Burgasser at http: //pono.ucsd. edu/ adam/browndwarfs/spexprism.

\section{References}

Ackerman, A. S., \& Marley, M. S. 2001, ApJ, 556, 872

Allard, F., Hauschildt, P. H., Alexander, D. R., Tamanai, A., \& Schweitzer, A. 2001, ApJ, 556, 357

Andersen, A. C., \& Jørgensen, U. G. 1995, Meteoritics, 30, 480

Anderson, D. R., Gillon, M., Maxted, P. F. L., et al. 2010, A\&A, 513, L3

Anderson, D. R., Smith, A. M. S., Madhusudhan, N., et al. 2013, MNRAS, 430, 3422

Aringer, B., Jørgensen, U. G., \& Langhoff, S. R. 1997, A\&A, 323, 202

Aringer, B., Kerschbaum, F., \& Jørgensen, U. G. 2002, A\&A, 395, 915

Asplund, M., Gustafsson, B., Lambert, D. L., \& Rao, N. K. 2000, A\&A, 353, 287

Barman, T. S., Macintosh, B., Konopacky, Q. M., \& Marois, C. 2011, ApJ, 733, 65

Barton, E. J., Yurchenko, S. N., \& Jonathan, T. 2013, MNRAS, 434, 1469

Blackwell, D. E., Lynas-Gray, A. E., \& Smith, G. 1995, A\&A, 296, 217

Bohren, C. F., \& Huffman, D. R. 1983, Absorption and Scattering of Light by Small Particles (John Wiley \& Sons Ltd)

Borysow, A., Jørgensen, U. G., \& Fu, Y. 2001, J. Quant. Spectr. Rad. Transf., 68, 235

Bowler, B. P., Liu, M. C., \& Dupuy, T. J. 2010, ApJ, 710, 45

Brooke, J. S. A., Bernath, P. F., Schmidt, T. W., \& Bacskay, G. B. 2013, J. Quant. Spectr. Rad. Transf., 124, 11

Brooke, J. S. A., Bernath, P. F., Western, C. M., Hemert, C. v., \& Groenenboom, G. C. 2014a, J. Chem. Phys., 141, 054310

Brooke, J. S. A., Ram, R., Western, C. M., et al. 2014b, ApJS, 210, 23

Bruggeman, D. A. G. 1935, Ann. Phys., 416, 636

Burgasser, A. J. 2014, in Astron. Soc. India Conf. Ser., 1, 7

Burgasser, A. J., McElwain, M. W., Kirkpatrick, J. D., et al. 2004, AJ, 127, 2856

Burgasser, A. J., Liu, M. C., Ireland, M. J., Cruz, K. L., \& Dupuy, T. J. 2008, ApJ, 681, 579

Burgasser, A. J., Dhital, S., \& West, A. A. 2009, AJ, 138, 1563

Burrows, A., \& Sharp, C. M. 1999, ApJ, 512, 843

Burrows, A., Hubbard, W. B., \& Lunine, J. I. 1989, ApJ, 345, 939

Burrows, A., Ram, R. S., Bernath, P., Sharp, C. M., \& Milsom, J. A. 2002, ApJ, 577,986

Burrows, A., Dulick, M., Bauschlicher, C. W., et al. 2005, ApJ, 624, 988

Burrows, A., Sudarsky, D., \& Hubeny, I. 2006, ApJ, 640, 1063

Carlson, B. E., Rossow, W. B., \& Orton, G. S. 1988, J. Atm. Sci., 45, 2066

Chiu, K., Fan, X., Leggett, S. K., et al. 2006, AJ, 131, 2722

Cooper, C. S., Sudarsky, D., Milsom, J. A., Lunine, J. I., \& Burrows, A. 2003, ApJ, 586, 1320

Coppola, C. M., Lodi, L., \& Tennyson, J. 2011, MNRAS, 415, 487

Decin, L., \& Eriksson, K. 2007, A\&A, 472, 1041

Decin, L., Vandenbussche, B., Waelkens, C., et al. 2003, A\&A, 400, 709

Dominik, C., Sedlmayr, E., \& Gail, H.-P. 1993, A\&A, 277, 578

Dorschner, J., Begemann, B., Henning, T., Jäger, C., \& Mutschke, H. 1995, A\&A, 300, 503

Doughty, N. A., \& Fraser, P. A. 1966, MNRAS, 132, 267

Doughty, N. A., Fraser, P. A., \& McEachran, R. P. 1966, MNRAS, 132, 255

Doyle, R. O. 1968, ApJ, 153, 987

Fortney, J. J., Robinson, T. D., Domagal-Goldman, S., et al. 2016, ArXiv e-prints [arXiv: 1602.06305]

Gail, H.-P., \& Sedlmayr, E. 1988, A\&A, 206, 153

GharibNezhad, E., Shayesteh, A., \& Bernath, P. 2013, MNRAS, 432, 2043

Gibson, N. P., Aigrain, S., Pollacco, D. L., et al. 2010, MNRAS, 404, L114

Grevesse, N., Asplund, M., \& Sauval, A. J. 2007, Space Sci. Rev., 130, 105

Groh, J. H., Meynet, G., Georgy, C., \& Ekström, S. 2013, A\&A, 558, A131

Gustafsson, B. 1971, A\&A, 10, 187

Gustafsson, B., Bell, R. A., Eriksson, K., \& Nordlund, A. 1975, A\&A, 42, 407

Gustafsson, B., Edvardsson, B., Eriksson, K., et al. 2008, A\&A, 486, 951

Harris, G. J., Tennyson, J., Kaminsky, B. M., Pavlenko, Y. C., \& Jones, H. R. A. 2006, MNRAS, 367, 400

Harris, G. J., Larner, F. C., Tennyson, J., et al. 2008, MNRAS, 390, 143

Helling, C., \& Woitke, P. 2006, A\&A, 455, 325

Helling, C., Jørgensen, U. G., Plez, B., \& Johnson, H. R. 1996, A\&A, 315, 194

Helling, C., Oevermann, M., Lüttke, M. J. H., Klein, R., \& Sedlmayr, E. 2001, A\&A, 376, 194

Helling, C., Ackerman, A., Allard, F., et al. 2008a, MNRAS, 391, 1854

Helling, C., Woitke, P., \& Thi, W.-F. 2008b, A\&A, 485, 547 
Helling, C., Lee, G., Dobbs-Dixon, I., et al. 2016, MNRAS, 460, 855

Hill, V., Christlieb, N., Beers, T. C., et al. 2017, A\&A, 607, A91

Höfner, S., Jørgensen, U. G., \& Loidl, R. 1998, Ap\&SS, 255, 281

Hui-Bon-Hoa, A., LeBlanc, F., \& Hauschildt, P. H. 2000, ApJ, 535, L43

Irwin, A. W. 1981, ApJS, 45, 621

Jäger, C., Dorschner, J., Mutschke, H., Posch, T., \& Henning, T. 2003, A\&A, 408, 193

John, T. L. 1967, ApJ, 149, 449

Jørgensen, U. G., Johnson, H. R., \& Nordlund, A. 1992, A\&A, 261, 263

Jørgensen, U. G., Hammer, D., Borysow, A., \& Falkesgaard, J. 2000, A\&A, 361, 283

Jørgensen, U. G., Jensen, P., Sørensen, G. O., \& Aringer, B. 2001, A\&A, 372 249

Karzas, W. J., \& Latter, R. 1961, ApJS, 6, 167

Kirkpatrick, J. D., Looper, D. L., Burgasser, A. J., et al. 2010, ApJS, 190, 100

Kupka, F., Dubernet, M.-L., \& VAMDC Collaboration. 2011, Balt. Astron., 20, 503

Kurucz, R. L. 1970, SAO Spec. Rep., 309

Kurucz, R. L. 2011, Can. J. Phys., 89, 417

Lambert, D. L., Gustafsson, B., Eriksson, K., \& Hinkle, K. H. 1986, ApJS, 62, 373

Lee, G., Helling, C., Dobbs-Dixon, I., \& Juncher, D. 2015, A\&A, 580, A12

Lewis, J. S. 1969, Icarus, 10, 365

Ludwig, H.-G., Allard, F., \& Hauschildt, P. H. 2002, A\&A, 395, 99

Lunine, J. I., Hubbard, W. B., \& Marley, M. S. 1986, ApJ, 310, 238

Madhusudhan, N., \& Seager, S. 2009, ApJ, 707, 24

Madhusudhan, N., \& Seager, S. 2010, ApJ, 725, 261

Madhusudhan, N., \& Seager, S. 2011, ApJ, 729, 41

Markwardt, C. B. 2009, in Astronomical Data Analysis Software and Systems XVIII, ASP Conf. Ser., 411, 251

Masseron, T., Plez, B., Eck, S. V., et al. 2014, A\&A, 571, A47

Matrozis, E., Ryde, N., \& Dupree, A. K. 2013, A\&A, 559, A115

Mie, G. 1908, Ann. Phys., 330, 377

Mihalas, D. 1965, ApJS, 9, 321

Mihalas, D. 1978, Stellar Atmospheres (W. H. Freeman and Co.)

Nissen, P. E., Chen, Y. Q., Carigi, L., Schuster, W. J., \& Zhao, G. 2014, A\&A, 568, A25

Palik, E. D. 1985, Handbook of Optical Constants of Solids (Academic Press)
Peach, G. 1970, Mem. R. Astron. Soc., 73, 1

Plez, B. 1992, ApJS, 94, 527

Plez, B., van Eck, S., Jorissen, A., et al. 2003, in Modelling of Stellar Atmospheres, eds. N. Piskunov, W. W. Weiss, \& D. F. Gray, IAU Symp., 210, 2

Popovas, A. 2014, Master's thesis, University of Copenhagen

Posch, T., Kerschbaum, F., Fabian, D., et al. 2003, ApJS, 149, 437

Rayner, J. T., Toomey, D. W., Onaka, P. M., et al. 2003, Publ. Astron. Soc. Pac., 115,362

Rossow, W. B. 1978, Icarus, 36, 1

Rothman, L. S., Gordon, I. E., Barber, R. J., et al. 2010, J. Quant. Spectr. Rad. Transf., 111, 2139

Ryde, N., Lambert, D. L., Richter, M. J., \& Lacy, J. H. 2002, ApJ, 580, 447

Schmidt, S. J., West, A. A., Burgasser, A. J., Bochanski, J. J., \& Hawley, S. L. 2010, AJ, 139, 1045

Schwenke, D. W. 1998, Faraday Discuss., 109, 321

Sharp, C. M., \& Huebner, W. F. 1990, ApJS, 72, 417

Siqueira-Mello, C., Chiappini, C., Barbuy, B., et al. 2016, A\&A, 593, A79

Somerville, W. B. 1964, ApJ, 139, 192

Somerville, W. B. 1965, ApJ, 141, 811

Street, R. A., Fulton, B. J., Scholz, A., et al. 2015, ApJ, 812, 161

Tsuji, T. 1964, Ann. Tokyo Astron. Obs., 9, 1

Tsuji, T. 1973, A\&A, 23, 411

Tsuji, T. 2001, in Ultracool Dwarfs: New Spectral Types L and T, eds. H. R. A. Jones, \& I. A. Steele, 9

Tsuji, T., Ohnaka, K., Aoki, W., \& Nakajima, T. 1996, A\&A, 308, L29

Van Eck, S., Neyskens, P., Jorissen, A., et al. 2017, A\&A, 601, A10

Walker, R. 2014, http://www.ursusmajor.ch/downloads/ spectroscopic-atlas-5_0-english.pdf

Weck, P. F., Stancil, P. C., \& Kirby, K. 2003, J. Chem. Phys., 118, 9997

Wende, S., Reiners, A., Seifahrt, A., \& Bernath, P. F. 2010, A\&A, 523, A58

Witte, S., Helling, C., \& Hauschildt, P. H. 2009, A\&A, 506, 1367

Witte, S., Helling, C., Barman, T., Heidrich, N., \& Hauschildt, P. H. 2011, A\&A, 529, A44

Woitke, P., \& Helling, C. 2003, A\&A, 399, 297

Woitke, P., \& Helling, C. 2004, A\&A, 414, 335

Yadin, B., Veness, T., Conti, P., et al. 2012, MNRAS, 425, 34

Zeidler, S., Posch, T., \& Mutschke, H. 2013, A\&A, 553, A81 


\section{Appendix A: Included atoms and molecules}

The following atoms and molecules were included in the chemical equilibrium calculations in MARCS.

Atoms (38): H, He, Li, Be, B, C, N, O, F, Na, Mg, Al, Si, $\mathrm{P}, \mathrm{S}, \mathrm{Cl}, \mathrm{K}, \mathrm{Ca}, \mathrm{Sc}, \mathrm{Ti}, \mathrm{V}, \mathrm{Cr}, \mathrm{Mn}, \mathrm{Fe}, \mathrm{Ni}, \mathrm{Cu}, \mathrm{Ge}, \mathrm{Br}, \mathrm{Rb}, \mathrm{Sr}, \mathrm{Y}$, $\mathrm{Zr}, \mathrm{Nb}, \mathrm{I}, \mathrm{Ba}, \mathrm{La}, \mathrm{Ce}, \mathrm{Nd}$.

Molecules (210): $\mathrm{H}^{-}, \mathrm{H}_{2}, \mathrm{H}_{2} \mathrm{O}, \mathrm{OH}, \mathrm{CH}, \mathrm{CO}, \mathrm{CN}, \mathrm{C}_{2}, \mathrm{~N}_{2}$, $\mathrm{O}_{2}, \mathrm{NO}, \mathrm{NH}, \mathrm{C}_{2} \mathrm{H}_{2}, \mathrm{HCN}, \mathrm{C}_{2} \mathrm{H}, \mathrm{HS}, \mathrm{SiH}, \mathrm{C}_{3}, \mathrm{CS}, \mathrm{SiC}, \mathrm{SiC}_{2}$, NS, SiN, SiO, SO, $\mathrm{S}_{2}$, SiS, TiO, VO, ZrO, MgH, HF, $\mathrm{HCl}, \mathrm{CH}_{4}$, $\mathrm{CH}_{2}, \mathrm{CH}_{3}, \mathrm{NH}_{2}, \mathrm{NH}_{3}, \mathrm{C}_{2} \mathrm{~N}_{2}, \mathrm{C}_{2} \mathrm{~N}, \mathrm{CO}_{2}, \mathrm{~F}^{-}, \mathrm{AlF}, \mathrm{CaF}, \mathrm{CaF}_{2}$, $\mathrm{MgOH}, \mathrm{Al}_{2} \mathrm{O}, \mathrm{AlOH}, \mathrm{AlOF}, \mathrm{AlOCl}, \mathrm{NaOH}, \mathrm{Si}_{2} \mathrm{C}, \mathrm{SiO}_{2}, \mathrm{H}_{2} \mathrm{~S}$, $\mathrm{CS}_{2}, \mathrm{AlCl}, \mathrm{NaCl}, \mathrm{KCl}, \mathrm{KOH}, \mathrm{CaCl}, \mathrm{CaCl}_{2}, \mathrm{CaOH}, \mathrm{TiO}_{2}, \mathrm{VO}_{2}$, $\mathrm{LiH}, \mathrm{LiO}, \mathrm{LiF}, \mathrm{LiCl}, \mathrm{BeH}_{2}, \mathrm{BeO}, \mathrm{BeF}, \mathrm{BeCl}, \mathrm{BeCl}_{2}, \mathrm{BeOH}, \mathrm{BH}$, $\mathrm{BH}_{2}, \mathrm{BO}, \mathrm{B}_{2} \mathrm{O}, \mathrm{BS}, \mathrm{BF}, \mathrm{BCl}, \mathrm{HBO}, \mathrm{HBO}_{2}, \mathrm{C}^{-}, \mathrm{C}_{2}^{-}, \mathrm{C}_{2} \mathrm{H}_{4}, \mathrm{NO}_{2}^{-}$, $\mathrm{N}_{2} \mathrm{H}_{2}, \mathrm{~N}_{2} \mathrm{H}_{4}, \mathrm{CN}_{2}, \mathrm{C}_{4} \mathrm{~N}_{2}, \mathrm{NO}_{2}, \mathrm{NO}_{3}, \mathrm{~N}_{2} \mathrm{O}, \mathrm{N}_{2} \mathrm{O}_{4}, \mathrm{HNO}, \mathrm{HNO}_{2}$, $\mathrm{HNO}_{3}, \mathrm{HCNO}, \mathrm{O}^{-}, \mathrm{O}_{2}^{-}, \mathrm{OH}^{-}, \mathrm{CO}_{2}^{-}, \mathrm{C}_{2} \mathrm{O}, \mathrm{HCO}, \mathrm{H}_{2} \mathrm{CO}, \mathrm{F}_{2}, \mathrm{FO}$, $\mathrm{NaH}, \mathrm{NaO}, \mathrm{NaF}, \mathrm{MgO}, \mathrm{MgS}, \mathrm{MgF} \mathrm{MgF}_{2}, \mathrm{MgCl}, \mathrm{MgCl}_{2}, \mathrm{AlH}$, $\mathrm{AlO}, \mathrm{AlO}_{2}, \mathrm{AlS}, \mathrm{AlF}_{2}, \mathrm{AlCl}_{2}, \mathrm{SI}^{-}, \mathrm{SiH}_{4}, \mathrm{SiF}, \mathrm{SiF}_{2}, \mathrm{SiCl}, \mathrm{SiCl}_{2}$, $\mathrm{PH}, \mathrm{PH}_{2}, \mathrm{PH}_{3}, \mathrm{CP}, \mathrm{NP}, \mathrm{PO}, \mathrm{PO}_{2}, \mathrm{PS}, \mathrm{PF}, \mathrm{PF}_{2}, \mathrm{PCL}, \mathrm{COS}, \mathrm{SO}_{2}$, $\mathrm{S}_{2} \mathrm{O}, \mathrm{SO}_{3}, \mathrm{Cl}^{-}, \mathrm{Cl}_{2}, \mathrm{CCl}, \mathrm{CCl}_{2}, \mathrm{CCl}_{3}, \mathrm{CCl}_{4}, \mathrm{ClO}, \mathrm{ClO}_{2}, \mathrm{Cl}_{2} \mathrm{O}$, $\mathrm{SCl}, \mathrm{SCl}_{2}, \mathrm{HClO}, \mathrm{CClO}, \mathrm{KH}, \mathrm{KO}, \mathrm{KF}, \mathrm{CaO}, \mathrm{CaS}, \mathrm{TiF}, \mathrm{TiF}_{2}$, $\mathrm{TiCl}, \mathrm{TiCl}_{2}, \mathrm{VN}, \mathrm{CrN}, \mathrm{CrO}, \mathrm{CrO}_{2}, \mathrm{FeO}, \mathrm{FeS}, \mathrm{FeF}, \mathrm{FeF}_{2}, \mathrm{FeCl}$, $\mathrm{FeCl}_{2}, \mathrm{NiCl}, \mathrm{CuO}, \mathrm{CuF}, \mathrm{CuCl}, \mathrm{SrO}, \mathrm{SrS}, \mathrm{SrF}, \mathrm{SrF}_{2}, \mathrm{SrCl}, \mathrm{SrCl}_{2}$, $\mathrm{SrOH}, \mathrm{ZrH}, \mathrm{ZrN}, \mathrm{ZrO}_{2}, \mathrm{ZrF}, \mathrm{ZrF}_{2}, \mathrm{ZrCl}, \mathrm{ZrCl}_{2}, \mathrm{HI}, \mathrm{BaO}, \mathrm{BaS}$, $\mathrm{BaF}, \mathrm{BaF}_{2}, \mathrm{BaCl}, \mathrm{BaCl}_{2}, \mathrm{BaOH}, \mathrm{NBO}, \mathrm{C}_{4}, \mathrm{C}_{5}, \mathrm{TiH}, \mathrm{CaH}, \mathrm{FeH}$, $\mathrm{CrH}$.

\section{Appendix B: Synthetic spectra decomposition}

We provide a detailed decomposition of the gas-contributions in Figs. B.1-B.3. At the shortest wavelengths, $\mathrm{SiO}, \mathrm{H}_{2}$, and $\mathrm{CO}$ are all very strong absorbers, with $\mathrm{SiO}$ being the most influential from $1.8-3 \mu \mathrm{m}$. $\mathrm{OH}$ also has a relatively strong absorption from $2.6-3.2 \mu \mathrm{m}$, but it is obscured by the $\mathrm{SiO}$ absorption. $\mathrm{NH}$ makes a short appearance around $3.4 \mu \mathrm{m}$. TiO absorption starts to grow from $4 \mu \mathrm{m}$ and completely dominates the spectrum from 4.4-9 $\mu \mathrm{m}$, with a few exceptions; at $7.5 \mu \mathrm{m}$, and $8.8 \mu \mathrm{m}$, the absorption of $\mathrm{TiO}$ weakens but is compensated for by the absorption of $\mathrm{VO}$ and $\mathrm{CrH}$, respectively. In fact, if there had been no $\mathrm{TiO}$ in the atmosphere, the metallic hydrides would have provided most of the absorption from $0.4-1.1 \mu \mathrm{m}$ with $\mathrm{CaH}$ peaking at $0.68 \mu \mathrm{m}, \mathrm{CrH}$ at $0.88 \mu \mathrm{m}$ and $1 \mu \mathrm{m}, \mathrm{FeH}$ at $1 \mu \mathrm{m}, \mathrm{MgH}$ at $0.51 \mu \mathrm{m}, \mathrm{SiH}$ at $0.42 \mu \mathrm{m}$ and $\mathrm{TiH}$ at $0.53 \mu \mathrm{m}$. $\mathrm{ZrO}$ also shows its strongest absorption in this region. Finally, $\mathrm{H}_{2} \mathrm{O}$ absorption shows up at $1.1 \mu \mathrm{m}$ and completely dominates the spectrum in the infrared and beyond. $\mathrm{LiH}$ and $\mathrm{NO}$ absorption both have a negligible effect on the spectrum because of their very low partial pressures. Even though the absorption coefficient of $\mathrm{CO}_{2}$ is larger than that of $\mathrm{CO}$ in the optical, the partial pressure of $\mathrm{CO}_{2}$ at these high temperatures is less than a thousandth of the partial pressure of $\mathrm{CO}$, and its spectroscopic features are therefore almost imperceptible. $\mathrm{CH}, \mathrm{C}_{2}, \mathrm{CN}$ and $\mathrm{HCN}$ are barely present in oxygen-rich atmospheres at these temperatures, and their contribution to the absorption is consequently negligible. These molecules will, however, become of interest if carbon is enhanced compared to the solar $\mathrm{C} / \mathrm{O}$ ratio. 
D. Juncher et al.: Atmosphere modeling with cloud formation
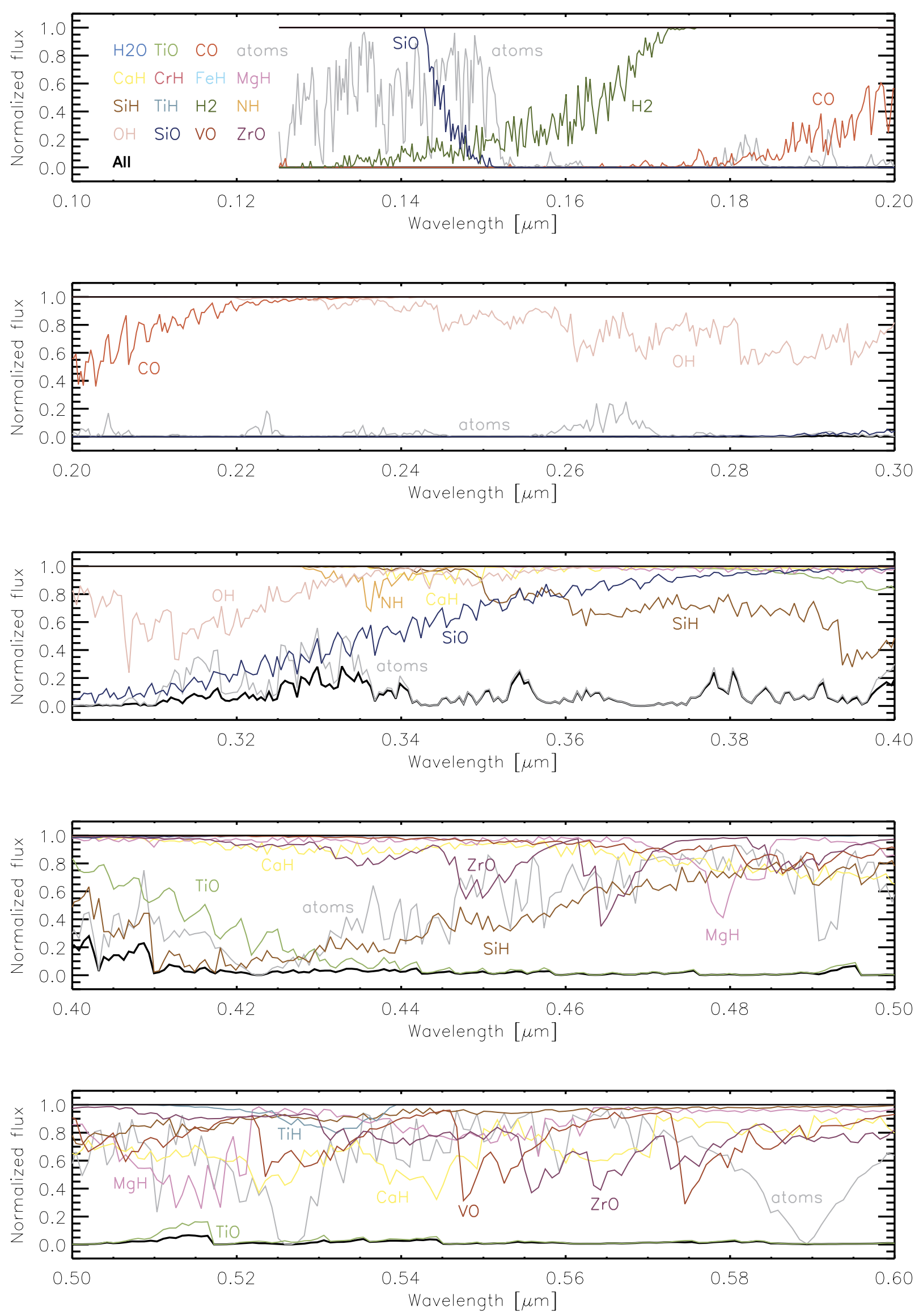

Fig. B.1. Spectral contributions of atomic and molecular opacity sources for a cloud-free MARCS-model atmosphere $\left(\right.$ of $T_{\text {eff }}=3000 \mathrm{~K}, \log (g)=$ 4.5 , solar element abundances) for the spectral range $\lambda=0.1-0.6 \mu \mathrm{m}$ (top to bottom panel). 
A\&A 608, A70 (2017)
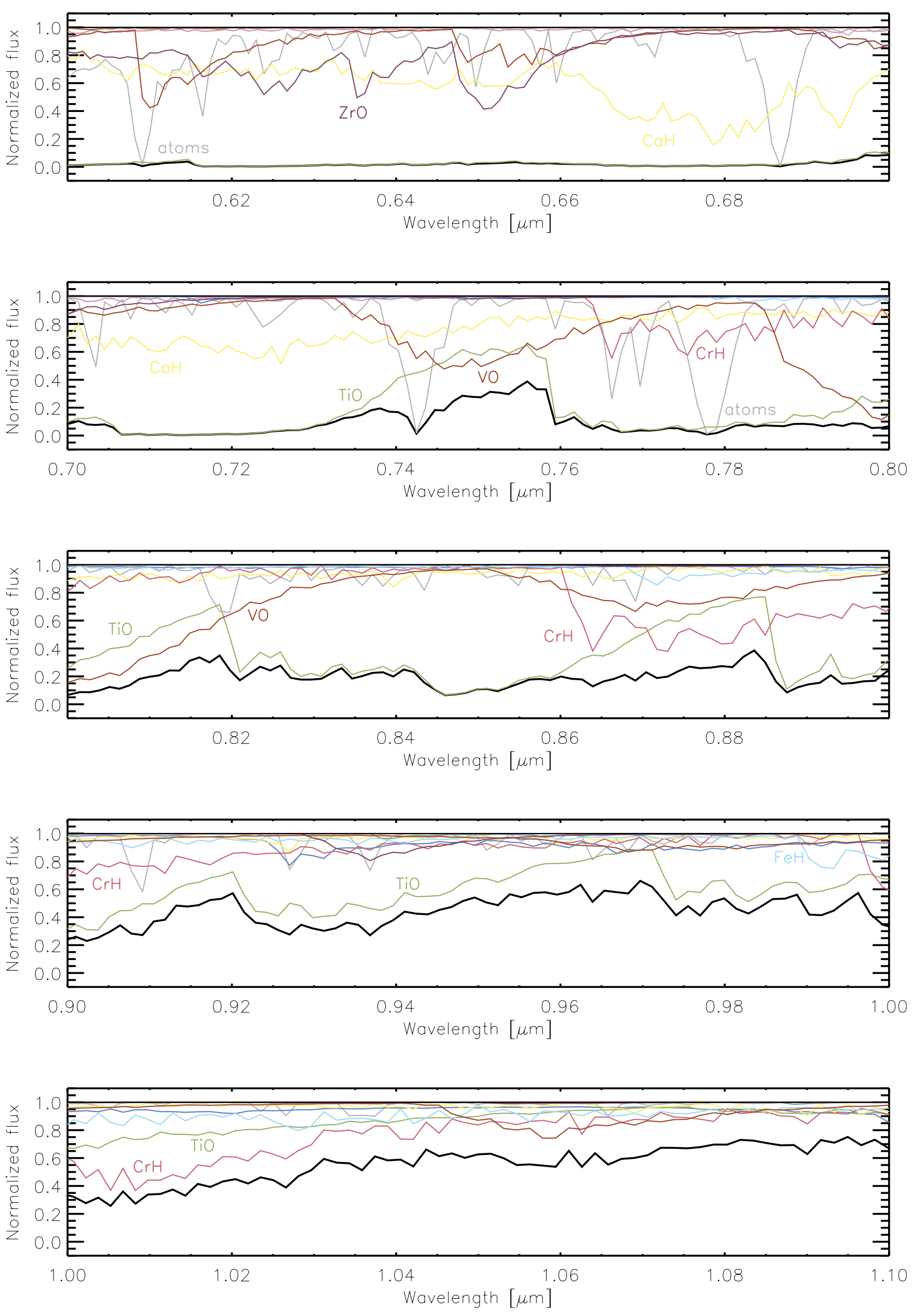

Fig. B.2. Same as Fig. B.1 but for $\lambda=0.6-1.1 \mu \mathrm{m}$ (top to bottom panel). 

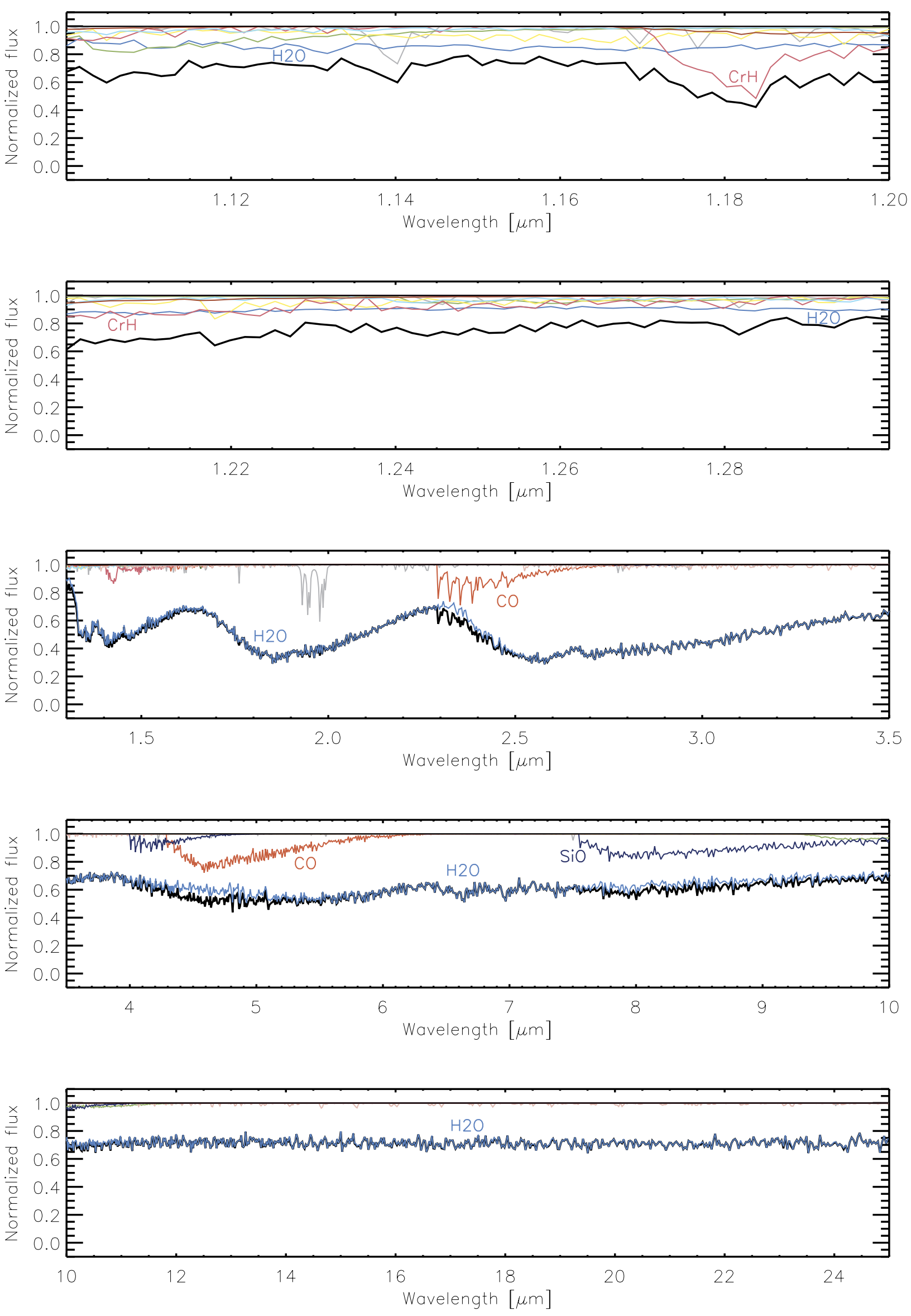

Fig. B.3. Same as Fig. B.1 but for $\lambda=1.1-24 \mu \mathrm{m}$ (top to bottom panel). 